\title{
Analysis of the Factors Affecting the Safety Performance in the Iranian Power Distribution Companies - Hybrid Approach of DEMATEL and ISM
}

Maryam Rezapour, M.Sc., Industrial Management Group, Shahed University, Tehran, Iran.

Saeed Safari, ( ${ }^{\star}$ Corresponding author), Associate Professor, Shahed University, Tehran, Iran. safari@shahed.ac.ir Alihossein Keshavarzi, Assistant Professor, Shahed University, Tehran, Iran.

\section{Abstract}

Background and aims: Workplace safety and protecting human and other resources is an important concern in the industry. Workplace accidents leads to damages to employees and affects business operations for a variety of reasons, including absenteeism, loss of productivity, and increased production costs. One of the most dangerous industries in the world is the electric power industry, and electric power has been identified as the fifth factor in terms of work accidents. The purpose of this study is to identify and classify the factors affecting the safety in power distribution companies and to determine the relationship between them.

Methods: Based on previous studies, 14 indicators affecting safety were identified in three dimensions: organizational, technical and human. Using the questionnaire, the rational relationship between the indicators is quantified and then, by combining DEMATEL and ISM, the influencing degree, influenced degree, centrality and causality between the indicators were calculated. In order to systematically analyze the relationship between the indicators, a hierarchical model was drawn using the ISM method; and based on the power of influence and dependence of indicators, the MICMAC diagram was drawn. According to the resultant model, the relationship between indicators were systematically analyzed.

Results: The top management commitment and safety standards as well as rules and regulations indicators, have the most power to influence other indicators and have a causal role; the risk identification and control, contractor and supplier management, incident analysis, safety audit, personal protective equipment, control and supervision, health and first aid, emergency response, safety training, communication, understanding the needs and expectations of the employees and other stakeholders, and developing a safety culture are the effective indicators.

Conclusion: This paper, in addition to introducing the theoretical basis for safety influencing indicators in power distribution companies and investigating their relationships, has proposed practical suggestions to prevent accidents and improve safety in the power distribution companies.

Conflicts of interest: None

Funding: None

\section{Keywords}

Safety indicators

Dematel

Interpretive structural

modeling

Power distribution companies

Received: 2019/12/8

Accepted : 2020/11/5 


\section{INTRODUCTION}

The rapid growth of technology, has caused substantial changes in the nature of the work and has increased the complexity of the systems in industries, and this has caused disastrous accidents in such industries [1].

One of the high-risk industries in the world is the electricity industry, so that electricity-related incidents in global studies, have been identified as the fifth affecting factor in the occurrence of accidents [2]. Since the most important capital of each society is its active human force, and it plays a pivotal role in the development and maintenance of the power distribution networks, it is necessary to identify and analyze the factors affecting the safety in this area, in order to reduce the accidents caused by electricity. This research has been conducted regarding the safety indices and the analysis of relations between them.

According to the literature review, there is little research on the safety of power distribution, and the effecting indicators of electrical safety are not comprehensively detected. In order to enrich the research indices, the other indices used in the similar researches have been used in the safety domain. In some researches, which have considered many effective indicators of safety in the electric power distribution companies, a suitable method for analyzing the data were not used.

According to literature reviews, 14 effective factors on safety in electricity distribution companies were identified. These factors which were based on Liu et al [3] classification, were classified in three main dimensions: organizational, technical and human. Therefore, the theoretical model of the research that was drawn according to the dimensions and safety indicators, is as follows (Fig. 1).

\section{METHODS}

The present study is an applied research in terms of purpose. In this research, by studying literature and background of the researches, the effective factors for safety in electricity distribution companies were identified, and after being reviewed by experts, the indicators were refined and finalized. Thus, in order to investigate the relationship between the indicators, a combination of DEMATEL and ISM was used. The Data collection tools including books, articles, Persian dissertations and e-databases were used to gather information about the background of the research and theoretical framework around the dimensions and

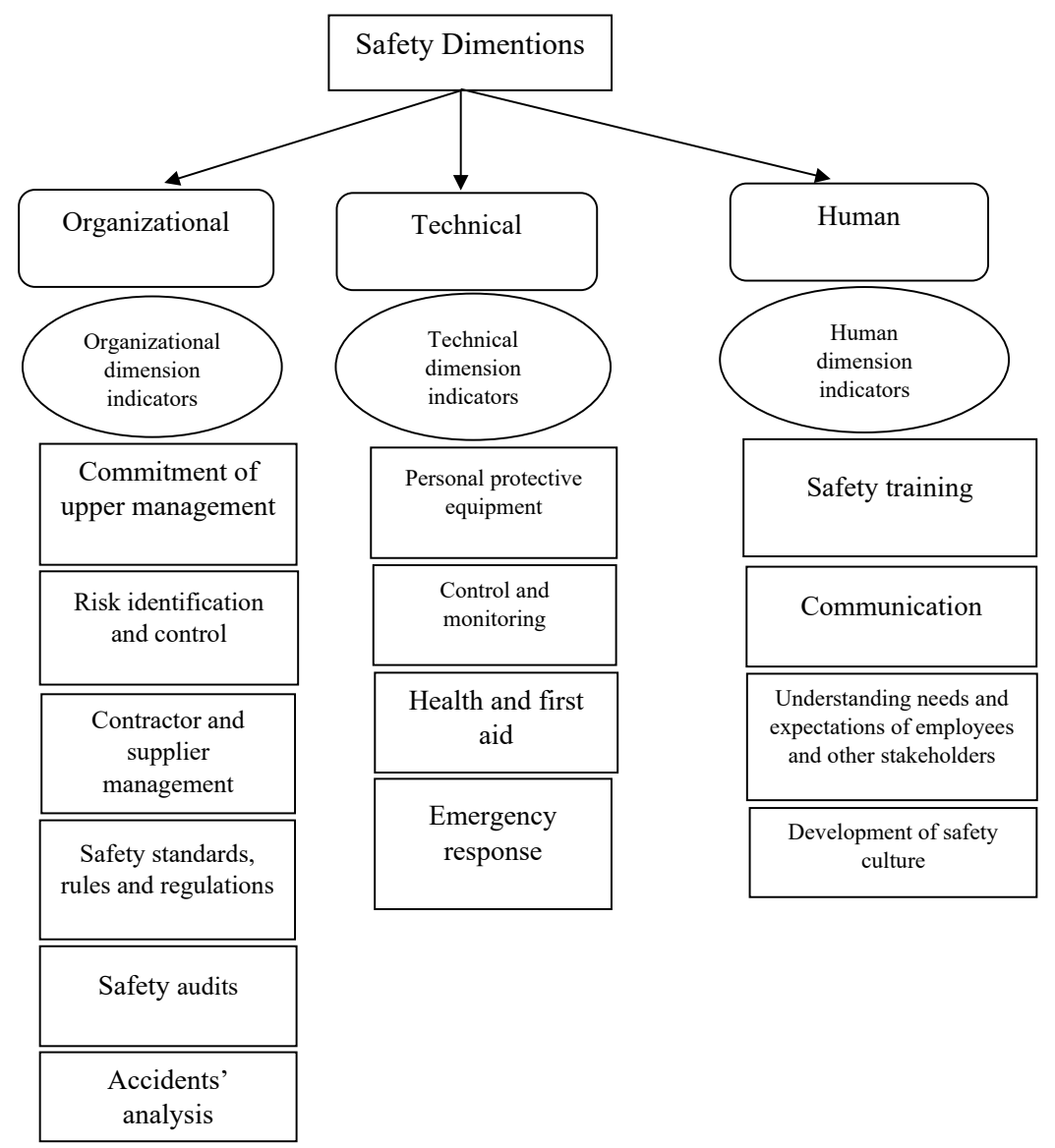

Fig. 1. Effective dimensions and indicators of safety in the power distribution companies 
Analysis of the Factors Affecting the Safety Performance...

effective factors on safety, and also a questionnaire was used to determine the effects between the effective indexes on safety in DEMATEL method.

The methodology used for analyzing the relationship between the effective indicators of safety, is borrowed from the study by Wang et al [4].

In the next stage, the effective indices were classified into four groups: independent, dependent, linkage and autonomous. The independent indicators, have a weak influence and weak correlation. The dependent indices have a weak influence, but highly dependent [5].

\section{RESULTS}

Based on the discussed topics in the previous chapters, in the DEMATEL method, the effect matrix was formed and the degree of influence, affectedness, and centrality were calculated. The degree of influence $\left(f_{i}\right)$, affectedness $\left(e_{i}\right)$, centrality $\left(m_{i}\right)$ and causality $\left(n_{i}\right)$ of the safety index $i(i=1,2,3, \ldots, 14)$, were calculated using the Excel software .

According to the levels of indexes and the reachability matrix, the final model was obtained as a hierarchical model of the indices, as well as the causal relationships between the indices in this model. As shown in Fig. 2, the final model consists of four levels; it should be noted that those effective factors on the safety, which are at higher levels, have more effectiveness and are slightly influenced by other indices. Top management commitment, standards, rules and regulations indicators, have the highest effect on the other indices. Also, according to the level of index, the fourth level indicators (top management commitment, safety standards, rules and regulations),

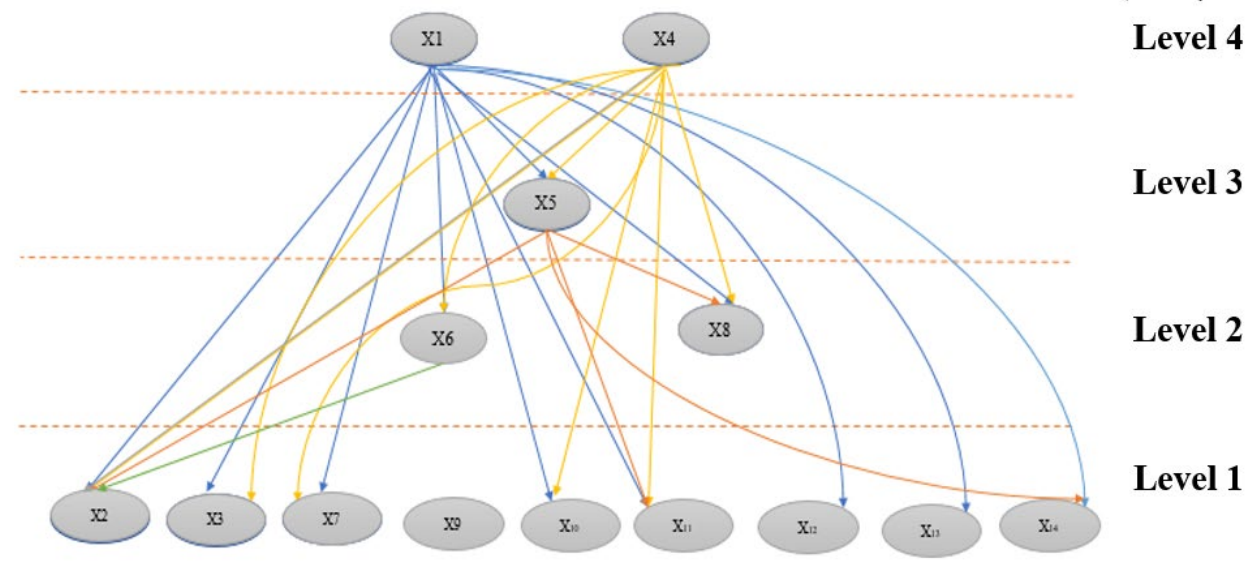

Fig. 2. Hierarchy of factors affecting safety

Table 1. The degree of effectiveness, affectedness, centrality and causation of criteria

\begin{tabular}{ccccccccc}
\hline indicator & $f_{i}$ & rank & $e_{i}$ & rank & $m_{i}$ & rank & $n_{i}$ & rank \\
\hline 1 & 2.71 & 1 & 1.79 & 8 & 4.50 & 1 & 0.92 & 3 \\
2 & 1.80 & 7 & 2.20 & 1 & 4 & 2 & -0.40 & 12 \\
3 & 1.70 & 9 & 1.76 & 9 & 3.46 & 9 & -0.05 & 5 \\
4 & 2.32 & 2 & 1.59 & 13 & 2.91 & 14 & 1.73 & 1 \\
5 & 2.01 & 3 & 1.75 & 10 & 3.76 & 7 & 1.41 & 2 \\
6 & 1.89 & 5 & 2 & 5 & 3.89 & 5 & -0.11 & 6 \\
7 & 1.56 & 11 & 1.98 & 6 & 3.55 & 8 & -0.42 & 13 \\
8 & 1.88 & 6 & 2.08 & 3 & 3.96 & 3 & -0.20 & 8 \\
9 & 1.34 & 13 & 1.70 & 12 & 3.04 & 13 & -0.35 & 11 \\
10 & 1.43 & 12 & 1.75 & 11 & 3.19 & 11 & -0.32 & 9 \\
11 & 1.75 & 8 & 2.10 & 2 & 3.86 & 6 & -0.34 & 10 \\
12 & 1.34 & 14 & 1.90 & 7 & 3.24 & 10 & -0.56 & 14 \\
13 & 1.58 & 10 & 1.57 & 14 & 3.15 & 12 & -0.002 & 4 \\
14 & 1.92 & 4 & 2.04 & 4 & 3.96 & 4 & 0.12 & 7 \\
\hline
\end{tabular}




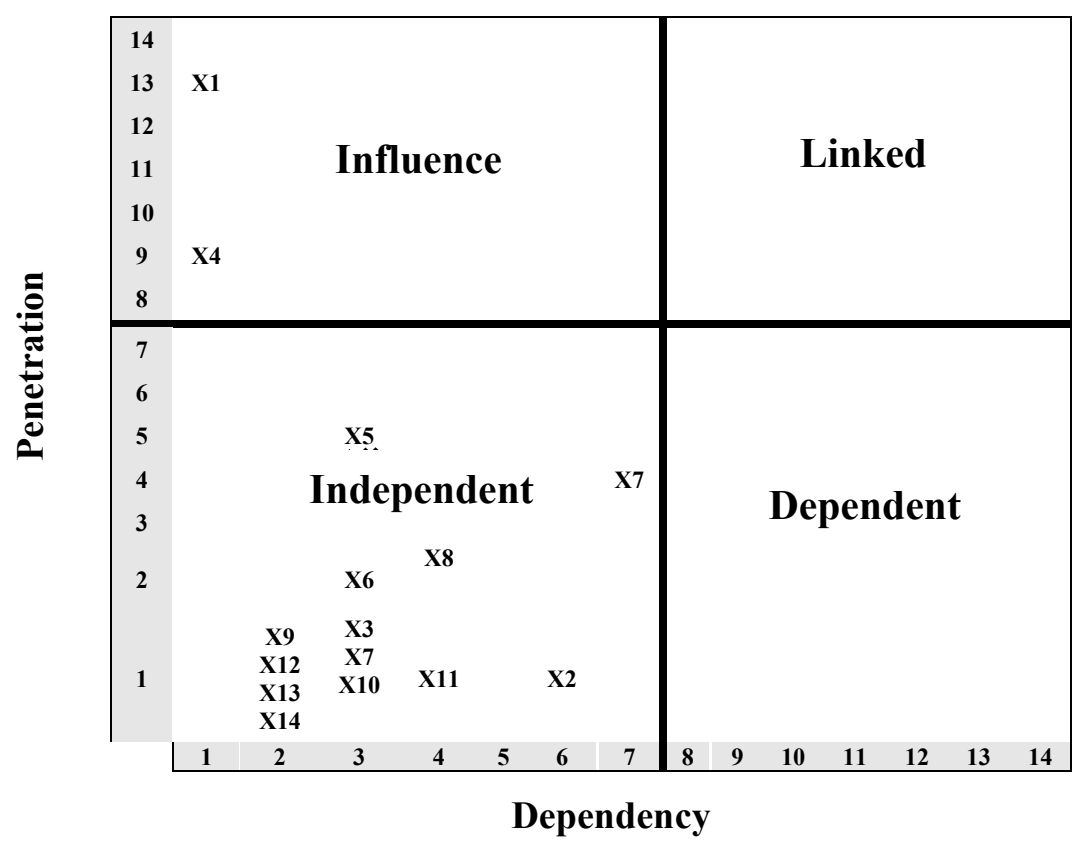

Fig. 3. penetration - dependency power curve

the third level ( analysis of accidents ) and the second level ( safety audits and control and monitoring), are all known as causal indicators and low level indices, first level (risk identification and control, contractors and suppliers management, personal protective equipment, health and first aid, emergency response , safety training, communication , understanding needs and expectations of employees and other stakeholders and the development of safety culture), are handicapped. Therefore, managers should pay more attention to the safety indicators that have the role of reason and should consider them in their decision making.

The effective indicators of safety, were classified based on their strength and influence on the four groups of independent, dependent, linkage and autonomous (Fig. 3). According to Fig. 3, the risk identification and control, contractors and supplier management, accidents analysis, safety audit, personal protective equipment, emergency response, safety training, communications, understanding needs and expectations of staff and other stakeholders and the development of safety culture in the category of independent indices, which have little leverage and dependency power, were classified. In addition, top management commitment, regulations and safety regulations indices were placed in the group of influential indicators that have a high penetration power and low dependency. Therefore, these two indices are considered to be two of the key variables.

\section{DISCUSSION}

This study has examined the effective factors on the safety of electric power distribution companies, using the hybrid method of DEMATEL and ISM. Based on previous studies, and the viewpoints of the safety experts, 14 effective indicators on the safety were identified, and were classified into three main categories, including: organizational, technical, and human indicators. This classification is compatible with the classification of liu et al [3].A DEMATEL questionnaire was used to collect the opinions of experts regarding the intensity of direct effect between the indicators. Using the combination of DEMATEL and ISM, the degrees of influence, effectiveness, centrality, and causality were calculated and then ranked.

The research findings showed that the indicators of organizational dimension had a higher impact on safety compared to the technical and human dimensions. This finding is compatible with the findings of other researchers, such as Tremblay et al [6]. However, our findings are inconsistent with Liu et al [3], Mazlina Zaira and Hadikusumo [7], who have declared that the technical dimension is the most important factor. Also, our findings are not compatible with the findings of Janackovic et al [8], which suggests that the human dimension is the most important factor, in terms of safety.

Among the organizational dimension indicators, the top management commitment and safety standards, rules and regulations are the most effective indices with the degrees of effectiveness of 2.71 and 2.32, and the causality degrees of 0.92 and 1.73 , respectively. These indicators have a causal role regarding other indicators. The management commitment has been investigated in previous researches, including: Nickpayam [9], and 
Analysis of the Factors Affecting the Safety Performance...

Wang et al [4]. Therefore, in order to introduce an effective and practical safety programs; support from top management is necessary to ensure any changes in other effective parameters of safety in organizations. The top management can play an important role in order to promote safety by defining the safety policy based on the company's safety management system, company's occupational health, long and short term goals and strategies, assigned responsibility and relevant roles to implement the safety system; and develop operational plans.

Also, the safety standards, rules and regulations should be clearly informed to all employees and other stakeholders of the company. Workplace accidents can be reduced significantly by severe supervision on implementation of rules in contracting matters, and purchasing equipment. The risk identification and control indicator with the centrality degree of 4 , was the second important safety indicator in electric power distribution companies.

\section{CONCLUSION}

On the basis of this finding, it is proper to identify the risks systematically and define appropriate corrective actions, then monitoring of their implementation. According to the high impact score of the accident analysis indicator, it is better to set a meeting with the presence of experts and related officials and analyze the accidents completely. Then the lessons learned from the analysis instructed to different units should be applied to business processes. Although this process exists in most power distribution companies, there is no proper mechanism for analyzing accidents and informing the results to other employees and most important of all, sending its reports to interested organizations, such as the Holding Company and Labor Office. The control and safety audit indices, by being placed at the third level, after the top management commitment, safety standards, rules and regulations and accident analysis, have the greatest impact on other indices. Thus, the proper control on the design process of networks and installations, locking facilities and land system of networks can greatly reduce risk of electric shocks to both company's staff and people and subscribers.

How to cite this article:

Maryam Rezapour, Saeed Safari, Alihossein Keshavarzi. Analysis of the Factors Affecting the Safety Performance in the Iranian Power Distribution Companies - Hybrid Approach of DEMATEL and ISM. Iran Occupational Health. 2021 (01 June);18:12. 

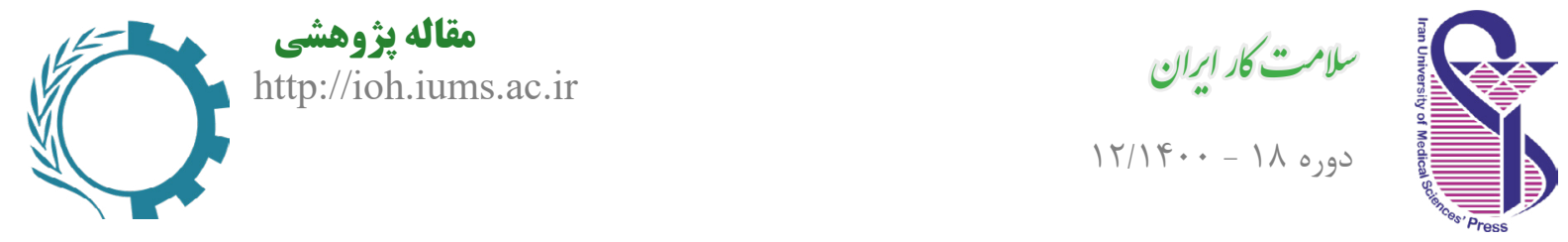

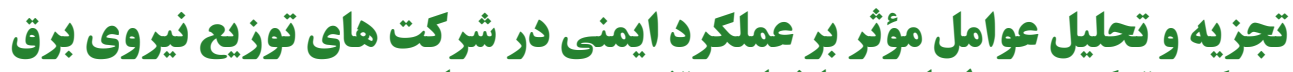

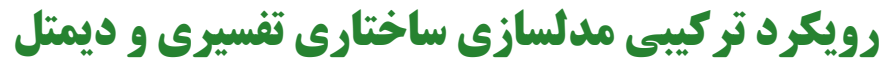

\author{
مريم رضايور: كارشناسى ارشد مديريت صنعتى، دانشعاه شاهد، تهران، ايران.

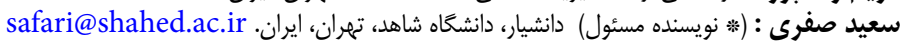

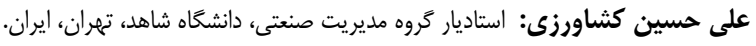

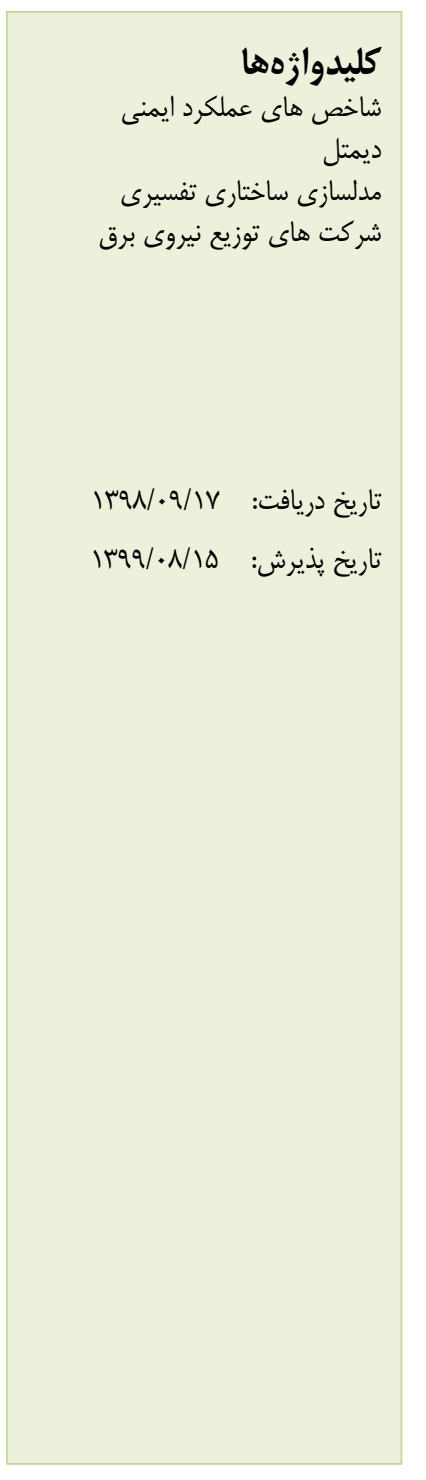

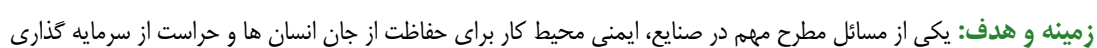

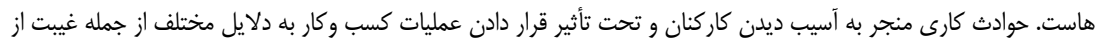

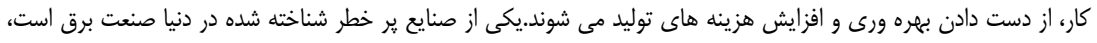

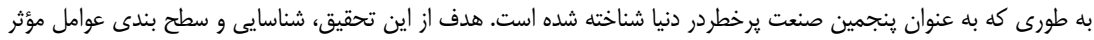

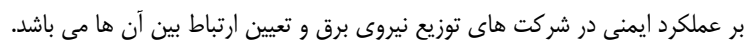

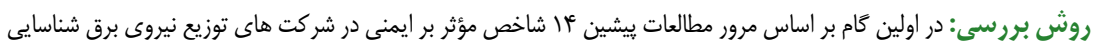

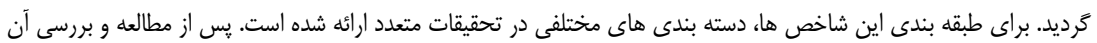

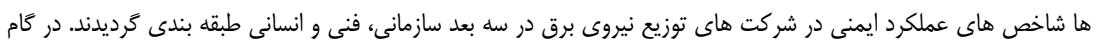

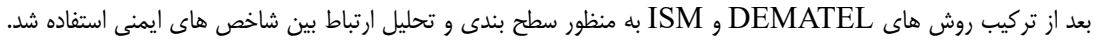

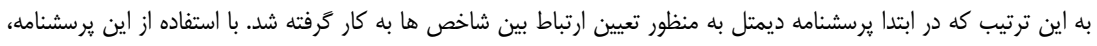

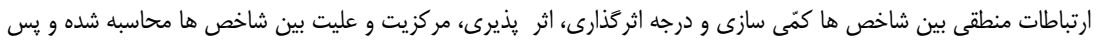

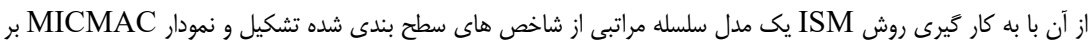

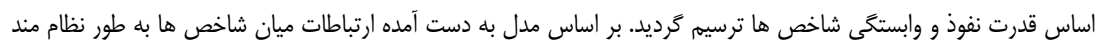

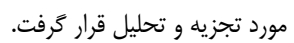

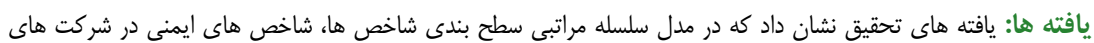

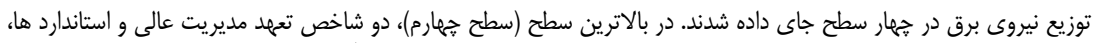

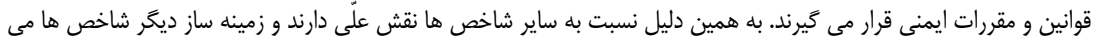
باشند. از سوى ديكر اين دو شاخص در نمر نمودار

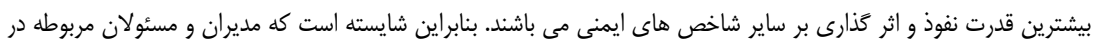

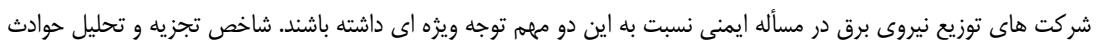

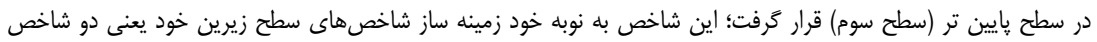

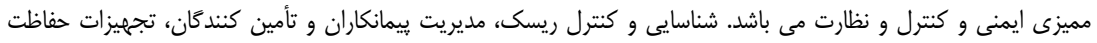

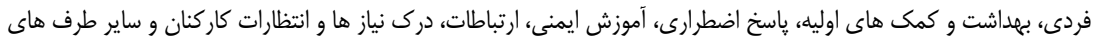

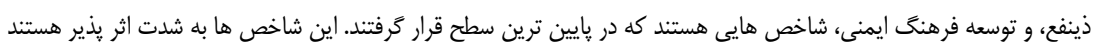
و نسبت به سايرين نقش معلول را دارند.

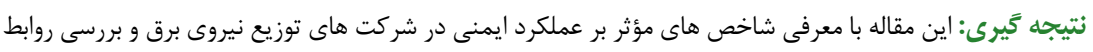

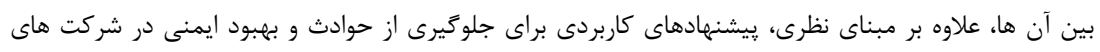
توزيع نيروى برق ارائه نموده است. تعارض منافع: كزارش نشده است. منبع حمايت كثنده: ندارد.

شيوه استناد به اين مقاله: Maryam Rezapour, Saeed Safari, Alihossein Keshavarzi. Analysis of the Factors Affecting the Safety Performance in the Iranian Power Distribution Companies - Hybrid Approach of DEMATEL and ISM. Iran Occupational Health. 2021 (01 June);18:12. 
يك شركت توزيع نيروى برق صربستان در جهار دسته

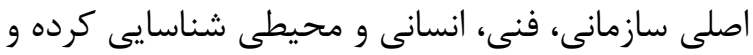

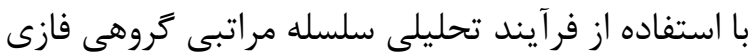

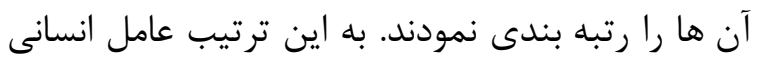

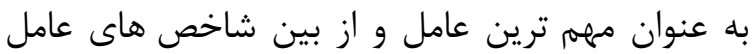

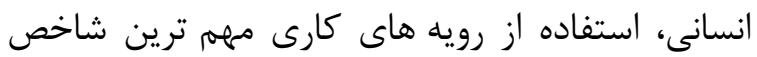

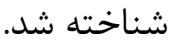

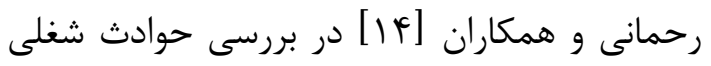

و علل آن در 9 ناحيه تحت يوشش ئران يك شركت توزيع

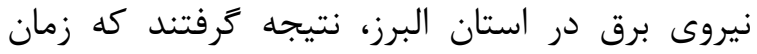

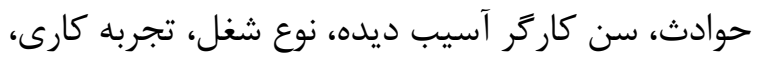

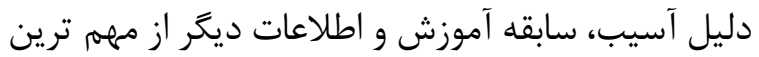

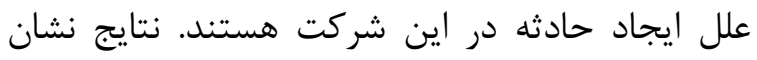

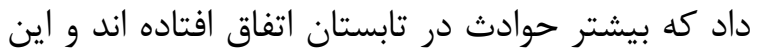

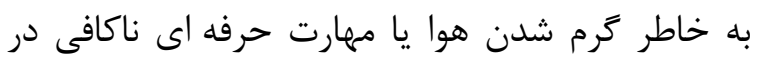

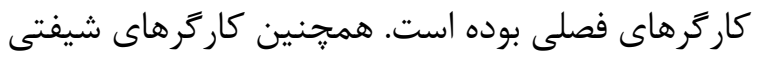

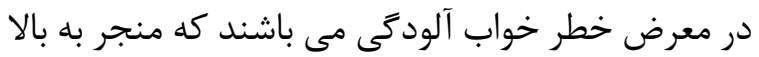

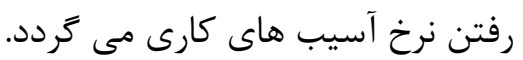

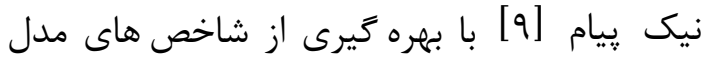
RSSB

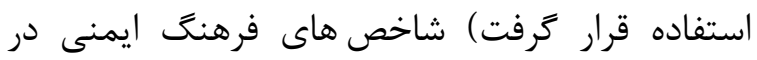

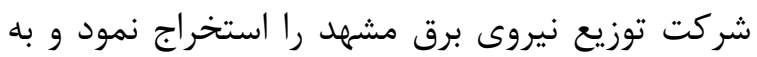

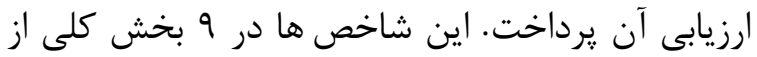

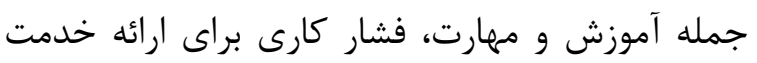

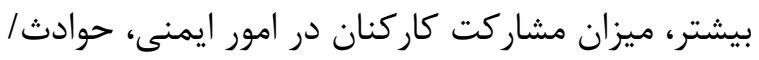

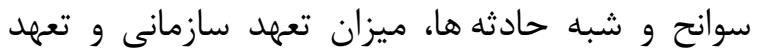

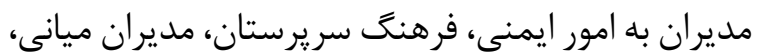

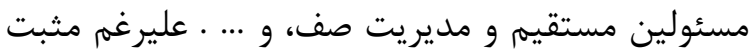

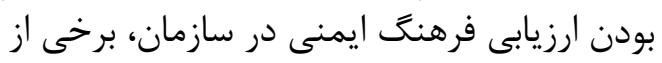

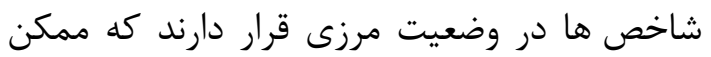

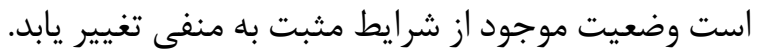

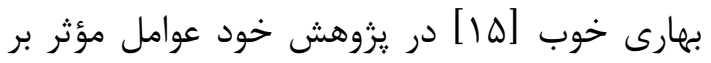

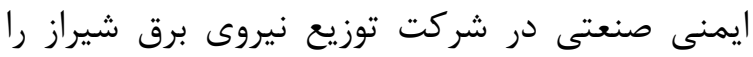

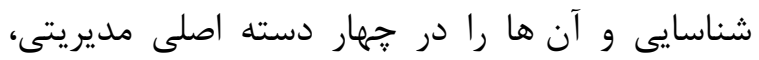

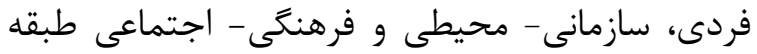

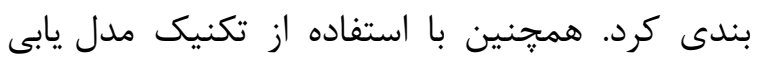

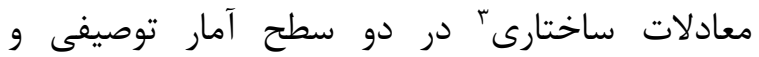

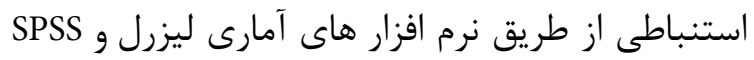

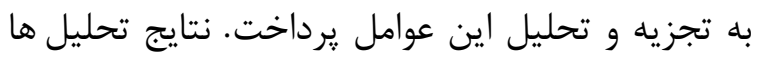

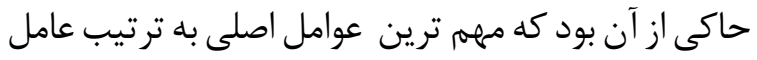

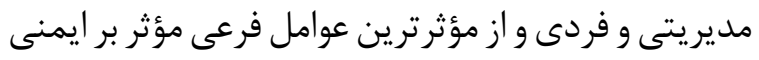

3-Structural Equation Model
مقامله

رشد سريع فناورى ها سبب ايجاد تغييرات اساسى

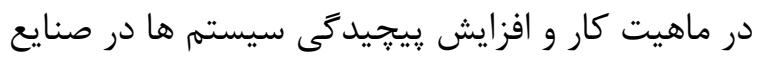

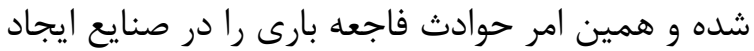

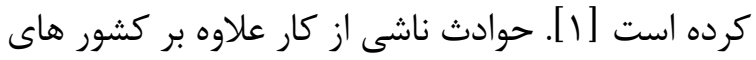
در حال توسعه در كشور هاى صنعتى نيز خسارات فراوان

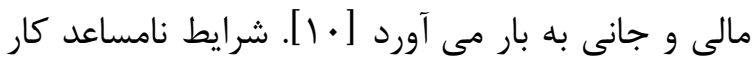
علاوه بر وقوع حوادث و بيمارى هاى نيروى به كارى كار، باعث

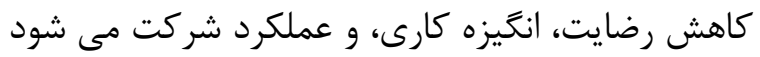

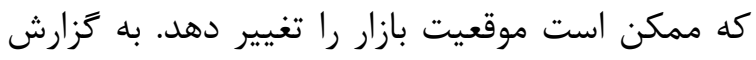

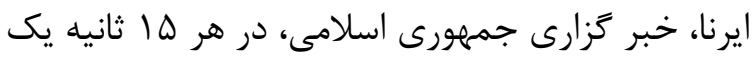

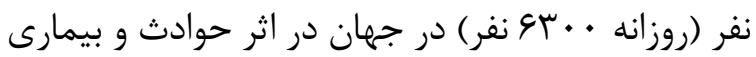

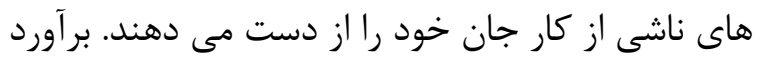

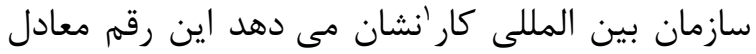

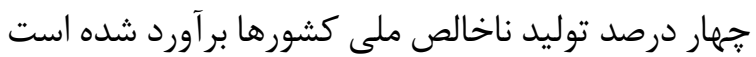

يكى از صنايع ير خطر شناخته شده در دنيا صنعت برق است، به طورى كه حوادث ناشى از برق در مطالعات

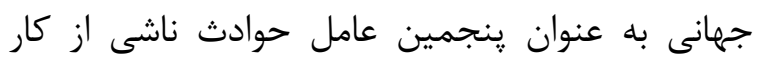

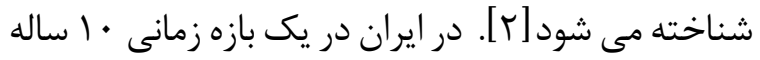

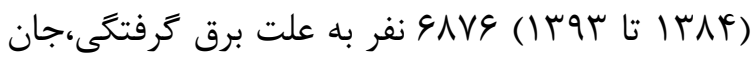

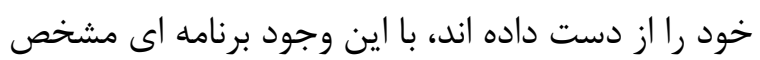

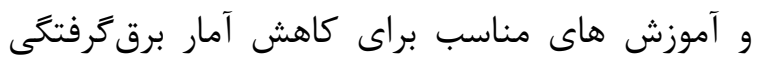

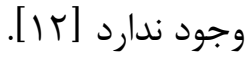

سيستم هاى مديريت ايمنى در حال حاضر به عنوران يك موضوع يزوهشى رايج است كه از يك ساختار اوليه

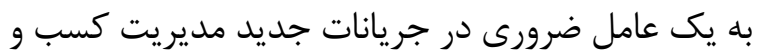

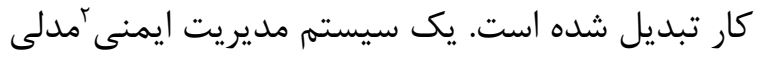
از مديريت است كه فعاليت ها، مسئوليت ها، شيوه ها،

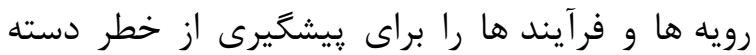

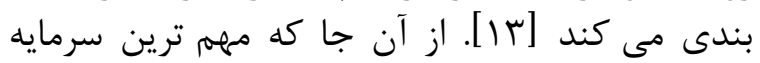

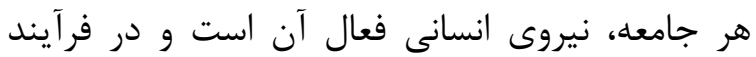

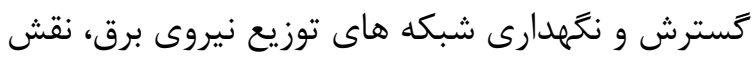

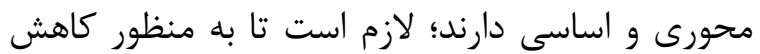

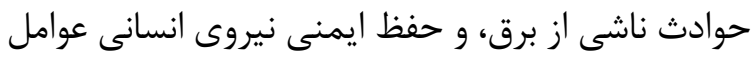

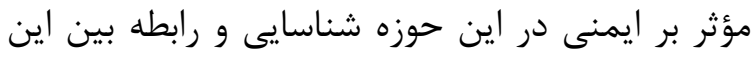

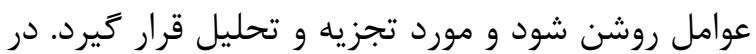

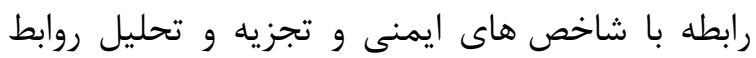

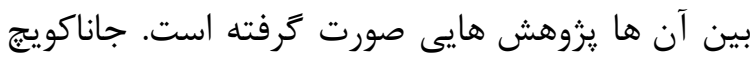

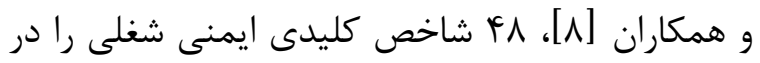

1- International Labour Organization 2-Safety Management System

Iran Occupational Health. 2021 (01 June);18: 12 


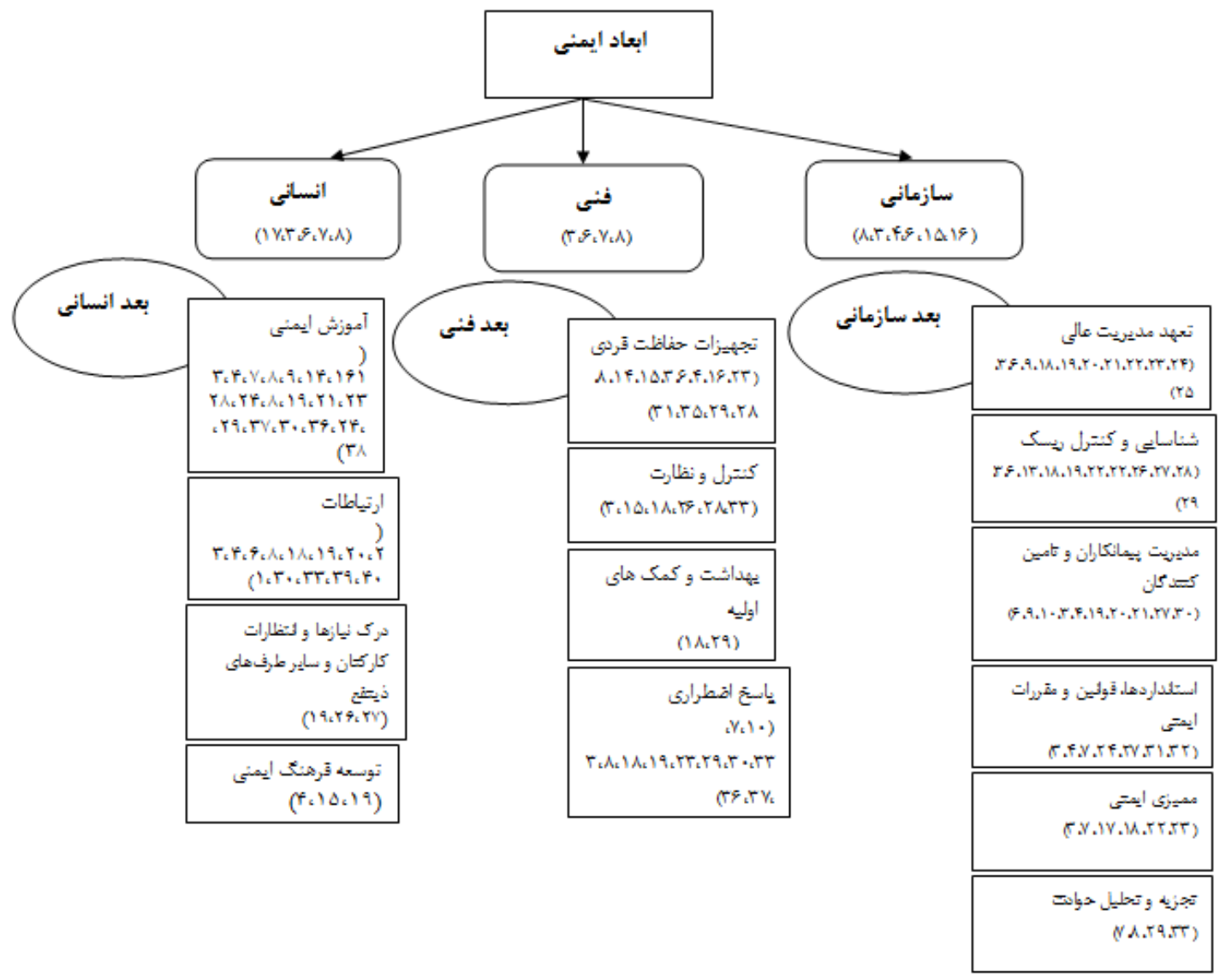

شكل ا. ابعاد و شاخص هاى مؤثر بر ايمنى در شركت هاى توزيع نيروى برق

شايستكى و فردى، ارزيابى اثربخشى آموزش و و ...)،

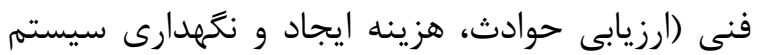

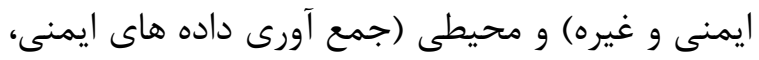

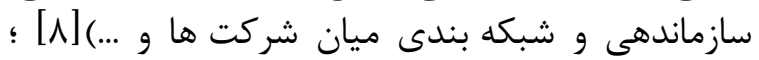

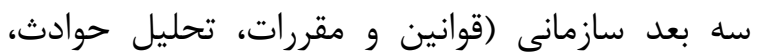

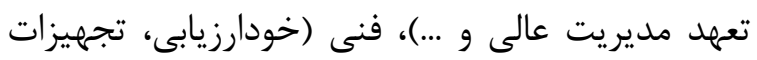

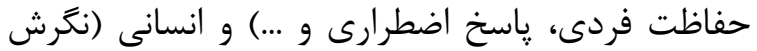

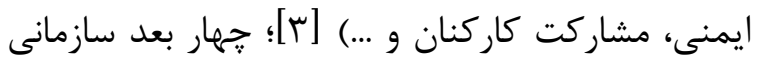

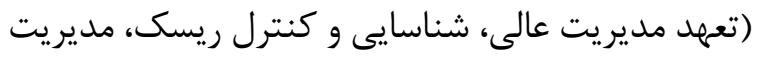

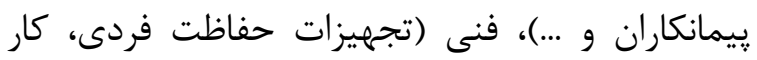

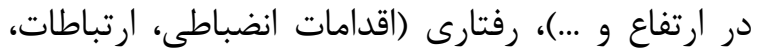

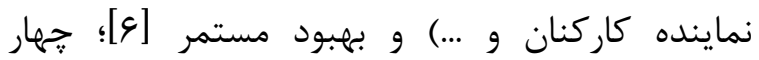

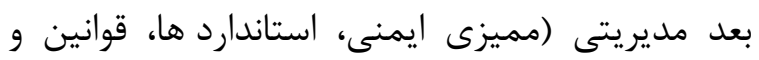

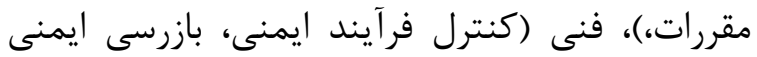

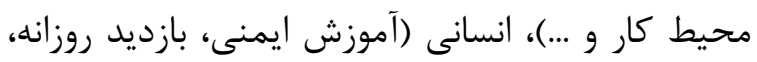

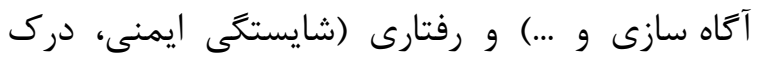

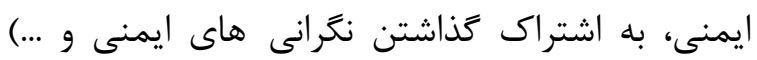

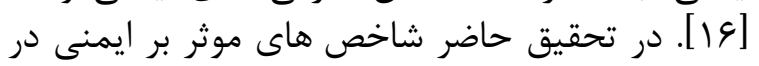

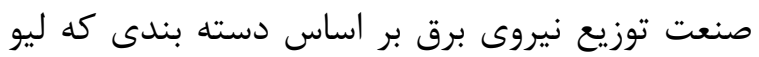

صنعتى برنامه ريزى، مديريت ايمنى و اطلاعات و فناورى

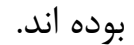
براساس بيشينه موجود، ملاحظه مى شود كه در حوزه

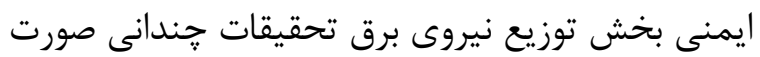

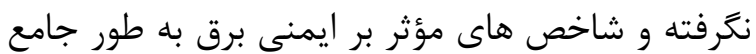

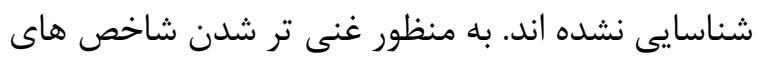

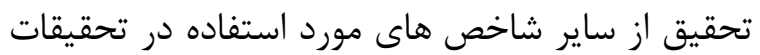

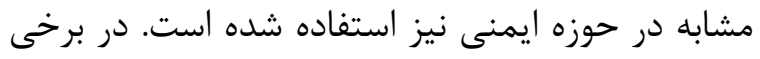

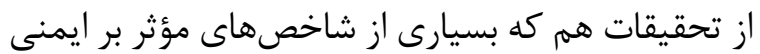

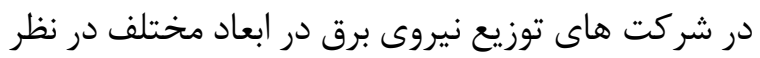

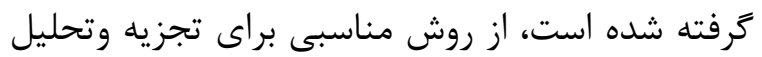

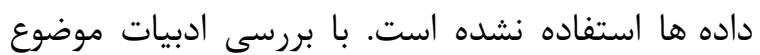

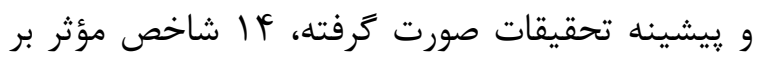

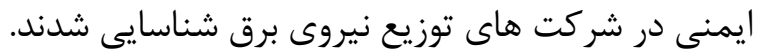

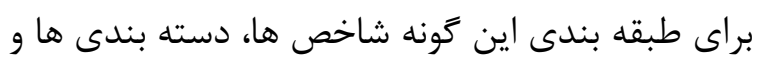

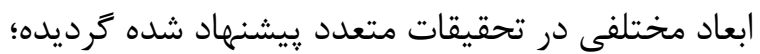

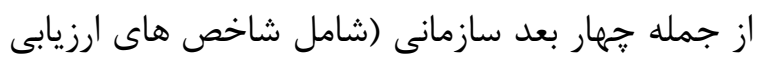

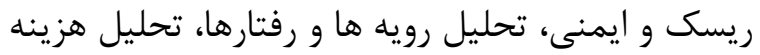

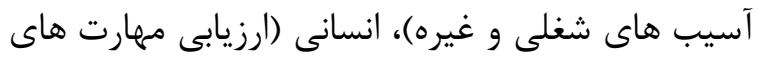


بر اساس طيف سه امتيازى ليكرت (r- إ-) نمره بدهند

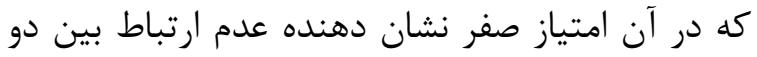

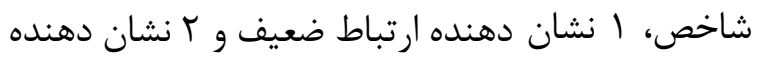
ارتباط قوى بين دو شاخص است.اتر نشان دهند أندان دهنده

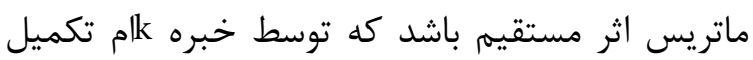

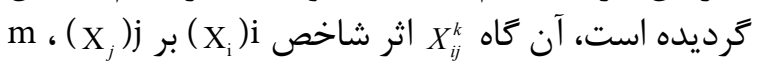

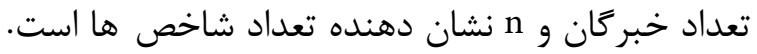

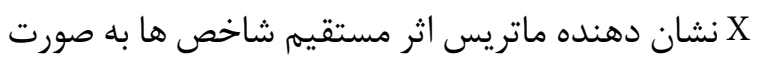

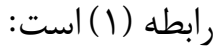

$$
X=\left[\begin{array}{ccc}
0 & \cdots & x_{1 n} \\
\vdots & \ddots & \vdots \\
x_{n 1} & \cdots & 0
\end{array}\right]=\left[x_{i j}\right]_{n \times n}
$$

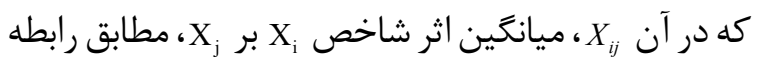

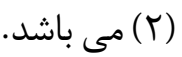

$$
x_{i j}=\left[\frac{1}{m} \sum_{k=1}^{m} x_{i j}^{k}+0.5\right](k=1,2, \ldots m)
$$

r- تشكيل ماتريس اثر جامع:

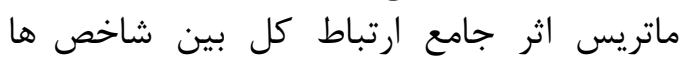

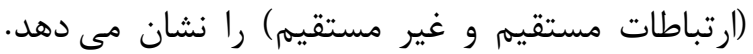

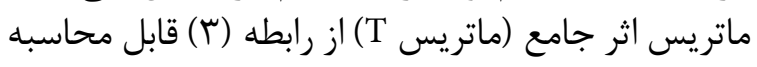

$$
\mathrm{T}=\mathrm{G}(I-G)^{-1}
$$

كه در آن G نرمال شده ماتريس اثر مستقيمم مىباشد و از رابطه (Y) قابل محاسبه است: نرمال

$$
\mathrm{G}=\frac{1}{\max _{1 \leqslant i \leqslant n} \sum_{j=1}^{n} x_{i j}} X
$$

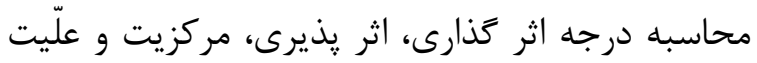

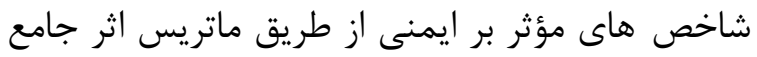
امكان يذير است.

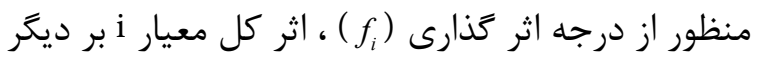
شاخص ها است. $f_{i}=\sum_{j=1}^{n} t_{i j}(i=1,2, \ldots n)$

درجه اثر يذيرى (e) اثر اثر كل ديخر شاخص ها بر معيار

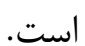

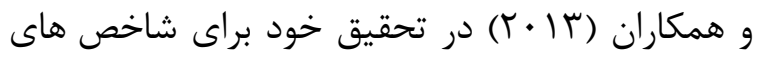

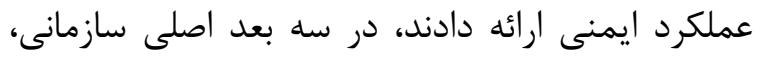

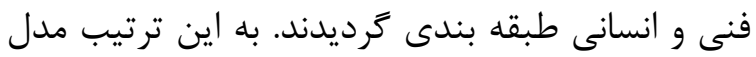

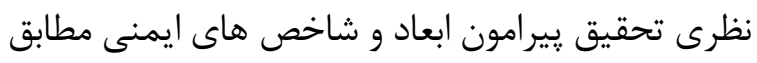
شكل ا ترسيم ترديد.

تحقيق حاضر به لحاظ هدف، از نوع كاربيق إندى مى بـ باشد،

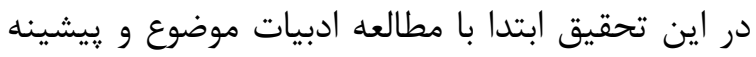

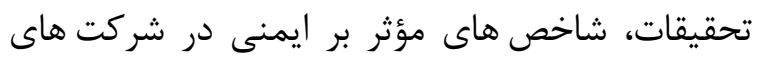

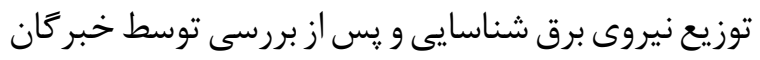

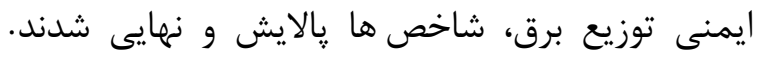

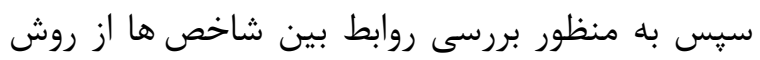

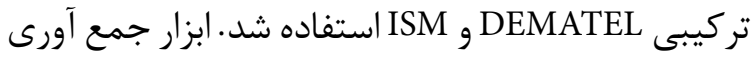

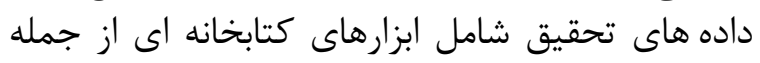

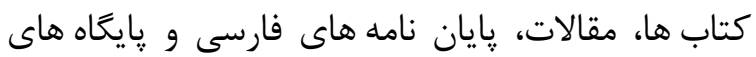

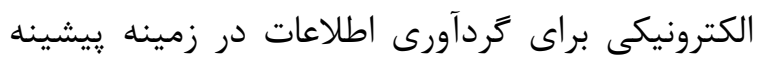

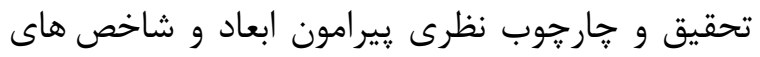

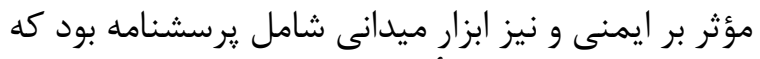

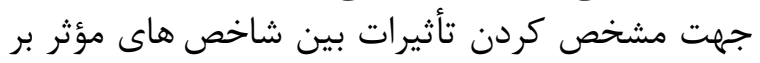

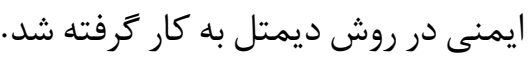

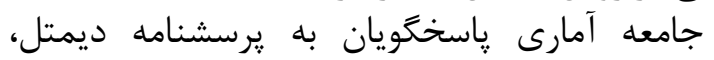

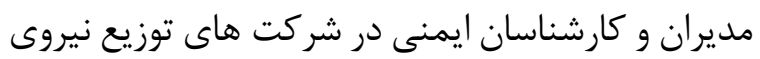

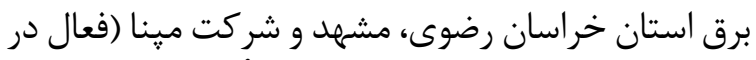

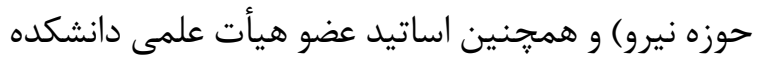

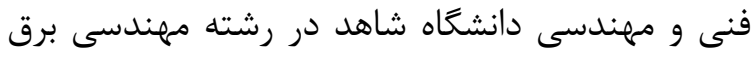

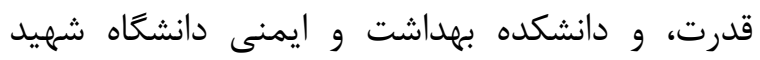

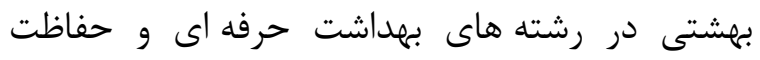

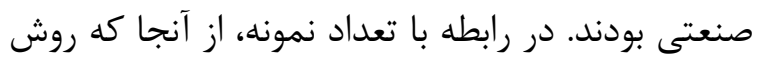

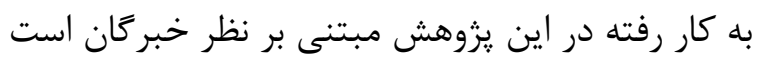

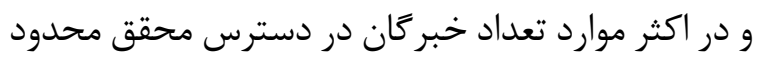

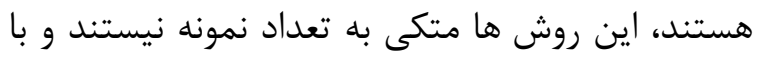

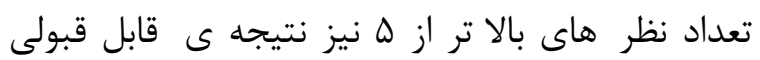

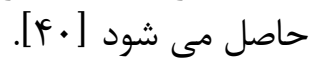

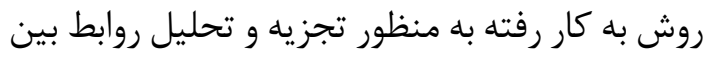

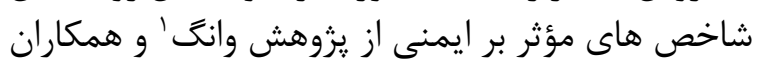

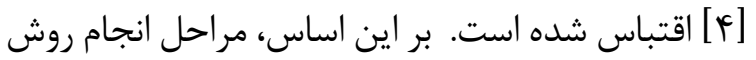

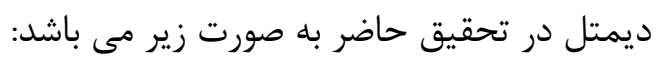

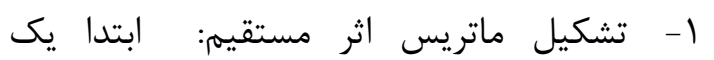

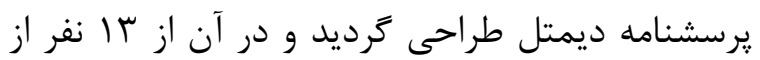

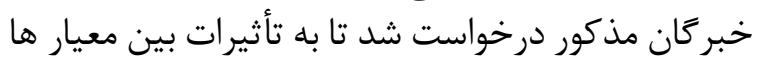

1-Wang

Iran Occupational Health. 2021 (01 June);18: 12 
نشان دهنده ارتباط ضعيف و يا عدم ارتباط بين دو معيار است.

$k_{i j}= \begin{cases}1, & k_{i j} \geqslant \lambda, i=1, \ldots, n \\ 0, & k_{i j}<\lambda, i=1, \ldots, n\end{cases}$

r- تشكيل مجموعه قابل دستيابى و مقدم و سطح

بندى شاخص ها

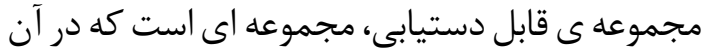

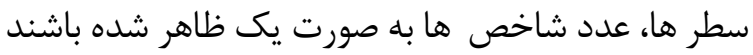

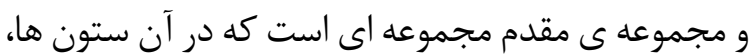

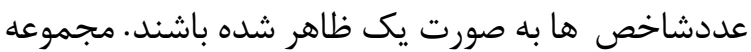

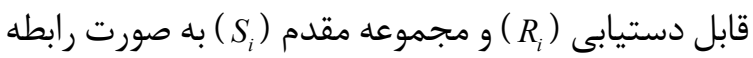

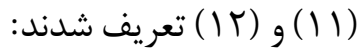

$R_{i}=\left\{x_{j} \mid x_{j} \in X, k_{i j}=1\right\},(i=1,2, \ldots, n)$

$S_{i}=\left\{x_{j} \mid x_{j} \in X, k_{j i}=1\right\},(i=1,2, \ldots, n)$

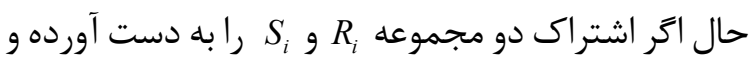
برابر مجموعه

$R_{i}=R_{i} \bigcap S_{i},(\mathrm{i}=1, \ldots, \mathrm{n})$

اين شاخص هادر سطح اول قرار مى گَيرند و همجنين

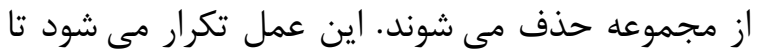
همه شاخص ها در سطوح بعدى قرار كيرند.

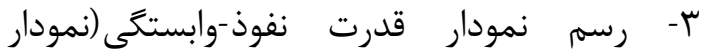

(MICMAC

در اين مرحله شاخص هاى مؤثر بر ايمنى در جهار

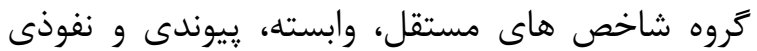

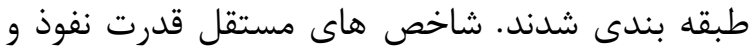

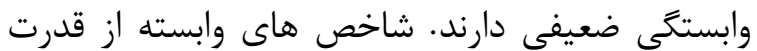

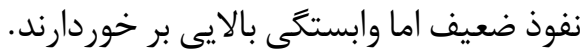

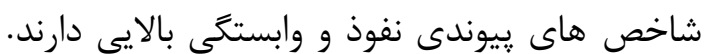

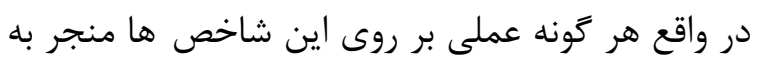

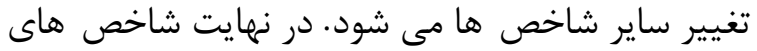

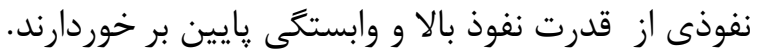

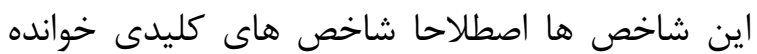

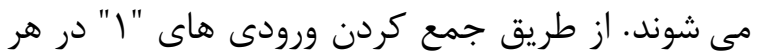

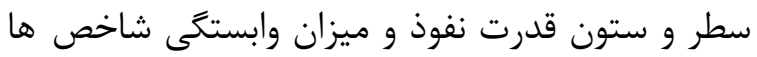

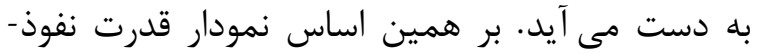
وابستكى ترسيم مى شود [هـ].

$$
e_{i}=\sum_{j=1}^{n} t_{j i}(i=1,2, \ldots n)
$$

درجه مركزيت معيار i (mi )، نشان دهنده اهميت آن آن شاخص در ميان كل شاخص هريت است

$$
m_{i}=f_{i}+e_{i}(i=1,2, \ldots, n)
$$

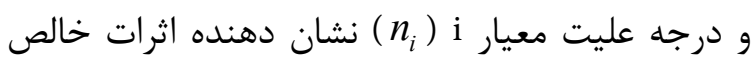
شاخص i بر ديخر شاخص هاميت معيار باشد.

$n_{i}=f_{i}-e_{i}(i=1,2, \ldots, n)$

$$
\text { ما - تشكيل ماتريس اثر كل }
$$

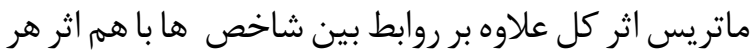

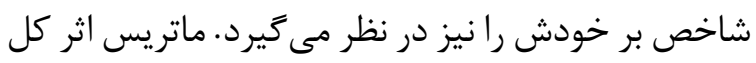
(H) از رابطه (9) قابل محاسبه است:

\section{$\mathrm{H}=\mathrm{T}+\mathrm{I}$}

تا اينجا روابط بين شاخص هاى مؤثر بر ايمنى و درجيه

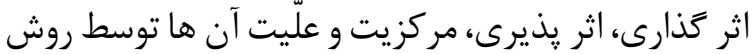

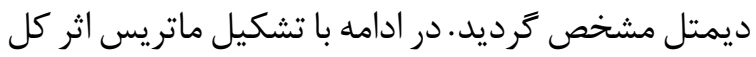

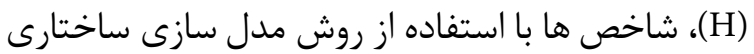

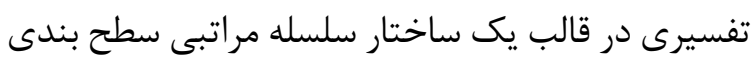

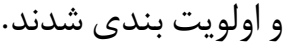

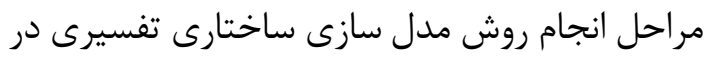

$$
\text { تحقيق حاضر به شرح زير است: }
$$

$$
\text { ا - تشكيل ماتريس دستيابى }
$$

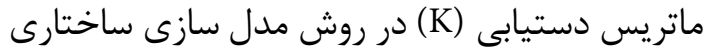

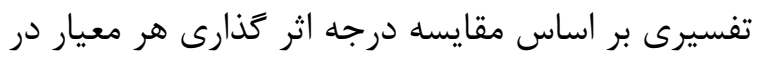

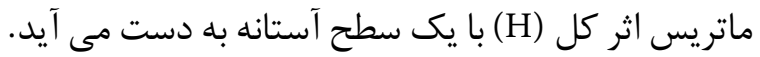

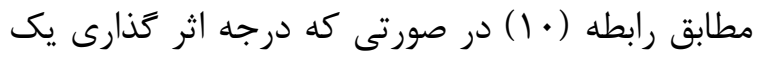

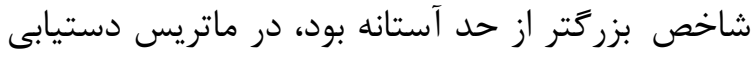

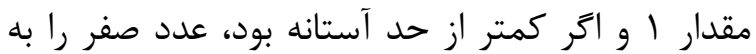

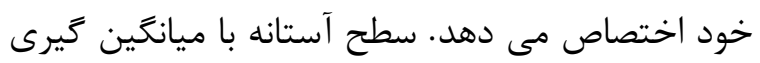

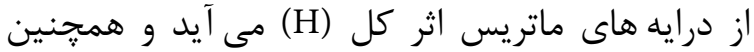

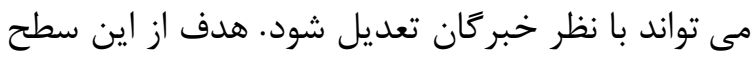

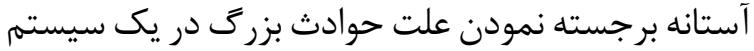

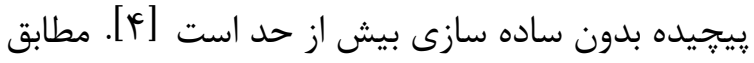

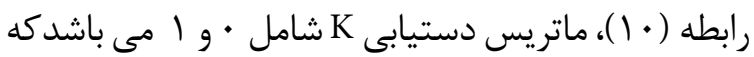

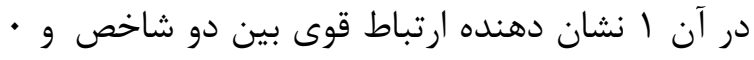


درايه هاى ماتريس اثر كل (H) به دست آمد و مقدار آن

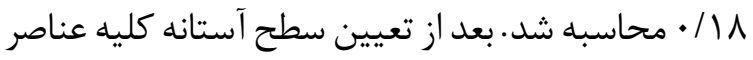

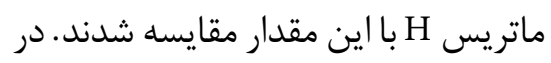

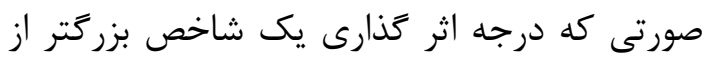

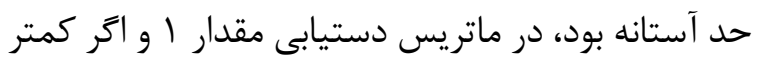

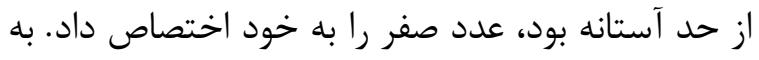

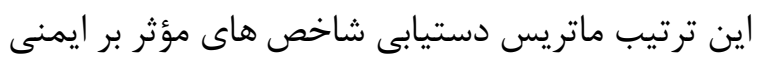
مطابق با جدول \& أشكيل شد.

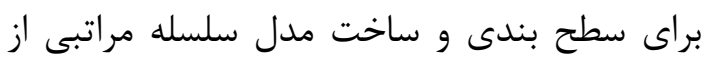

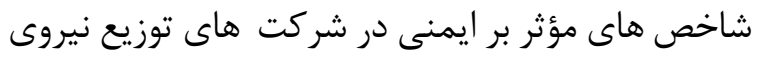

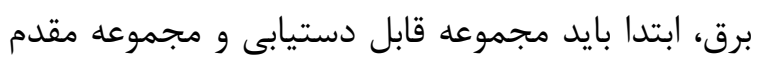
براى هر يك از شاخص ها تشكيل شود.

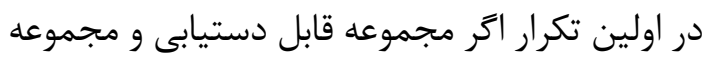

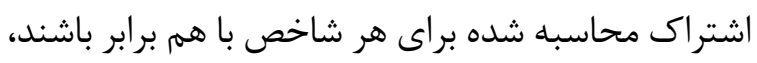

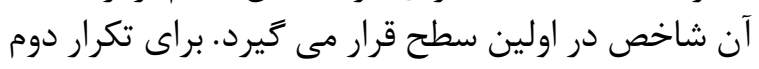

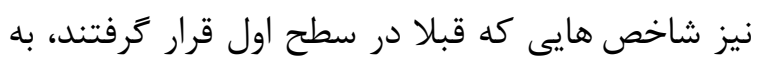

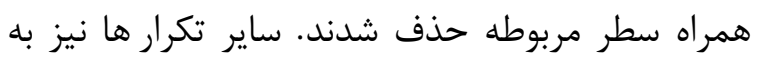
همين ترتيب صورت يذيرفت مرنه با توجه به سطوح شاخص ها ونورت ماتريس دستيابى، مدل
بافتنه ها

بر اساس مباحث مطرح شده در بخش هائ مائ قبل،

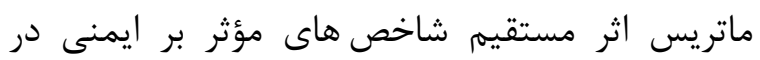

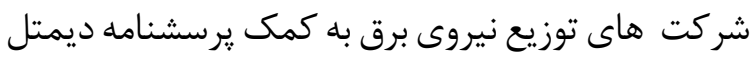

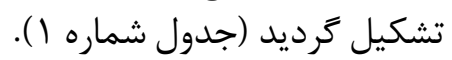

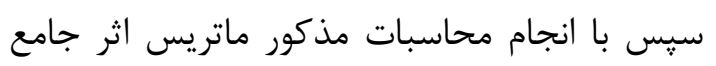

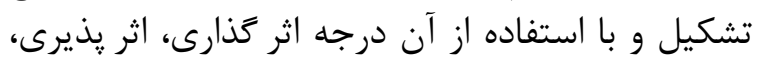

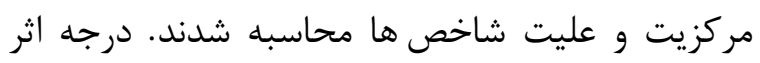

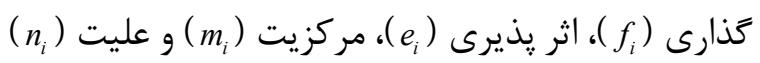

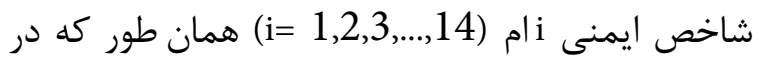

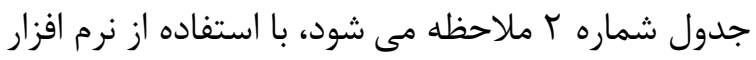
Excel محاسبه شده است.

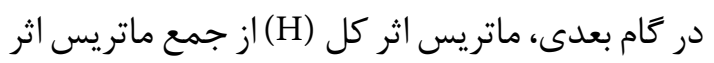

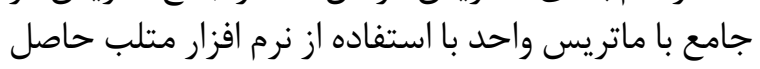

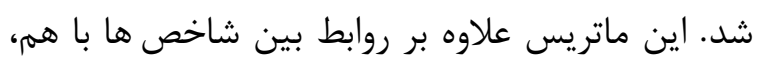

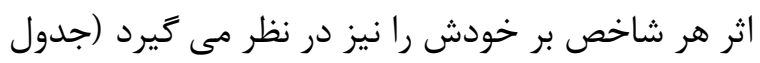
شماره ب).

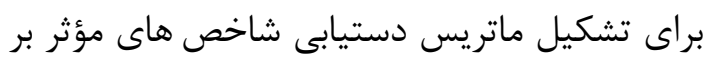

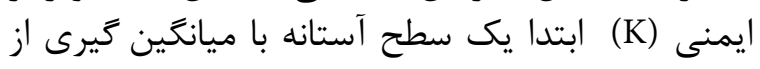

جدول ا. ماتريس اثر مستقيم شاخص هاى مؤثر بر ايمنى

\begin{tabular}{|c|c|c|c|c|c|c|c|c|c|c|c|c|c|c|}
\hline 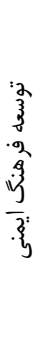 & 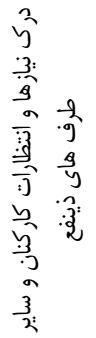 & $\begin{array}{l}\overline{3} . \overline{3} \\
\overline{3}\end{array}$ & $\frac{\sqrt{\alpha}}{3}$ & $\frac{\overrightarrow{3}}{\frac{3}{3}}$ & 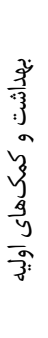 & 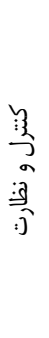 & 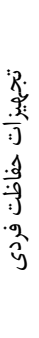 & 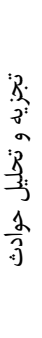 & $\begin{array}{c}\frac{z}{3} \\
y \\
\frac{y}{3} \\
y\end{array}$ & 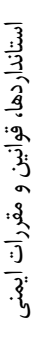 & 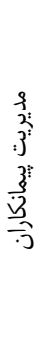 & 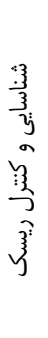 & $\begin{array}{l}2 \\
3 \\
1 \\
3 \\
3: \\
3 \\
3 \\
y \\
y \\
y\end{array}$ & شاخص \\
\hline r & r & r & r & r & r & r & r & r & r & 1 & r & r & · & تعهد مديريت عالى \\
\hline r & 1 & 1 & r & 1 & 1 & 1 & 1 & r & 1 & 1 & 1 & . & 1 & شناسايى و كنترل ريسك \\
\hline 1 & 1 & 1 & 1 & 1 & 1 & r & 1 & 1 & 1 & · & · & r & r & مديريت ييمانكاران \\
\hline 1 & 1 & 1 & r & r & 1 & r & r & r & r & . & r & t & 1 & مقرات استاردها، قوانين و \\
\hline r & 1 & 1 & r & 1 & 1 & r & r & 1 & . & 1 & 1 & r & 1 & مميزى ايمنى \\
\hline 1 & 1 & 1 & r & r & 1 & r & 1 & . & 1 & 1 & 1 & r & 1 & تجزيه و تحليل حوادث \\
\hline r & 1 & 1 & 1 & 1 & 1 & 1 & $\cdot$ & 1 & 1 & • & 1 & r & 1 & تجهيزات حفاظت فردى \\
\hline 1 & 1 & 1 & 1 & 1 & 1 & - & r & r & r & . & r & r & 1 & كنترل و نظارت \\
\hline 1 & 1 & 1 & 1 & 1 & · & 1 & 1 & 1 & 1 & . & 1 & 1 & 1 & بهداشت و كمكهاى \\
\hline 1 & 1 & r & 1 & . & 1 & 1 & 1 & 1 & 1 & . & 1 & 1 & 1 & ياسخ اضطرارى \\
\hline r & 1 & 1 & · & 1 & r & 1 & r & r & 1 & . & 1 & 1 & 1 & آموزش \\
\hline 1 & 1 & . & 1 & 1 & 1 & 1 & 1 & 1 & 1 & . & 1 & 1 & 1 & $\begin{array}{c}\text { درى نيازها و انتظارات } \\
\text { داطاطات }\end{array}$ \\
\hline 1 & $\cdot$ & r & 1 & 1 & 1 & 1 & 1 & 1 & 1 & - & 1 & 1 & r & كاركنان و ساير طرف \\
\hline - & 1 & r & r & 1 & 1 & r & 1 & 1 & 1 & 1 & 1 & 1 & r & توسعه فرهنگ ايمنى \\
\hline
\end{tabular}


جدول r. درجه اثركذارى، اثريذيرى، مركزيت و عليت معيارها

\begin{tabular}{|c|c|c|c|c|c|c|c|c|}
\hline رتبه بندى & $n_{i}$ & رتبه بندى & $m_{i}$ & رتبه بندى & $e_{i}$ & رتبه بندى & $f_{i}$ & شاخص \\
\hline r &.$/ 95$ & 1 & $F / Q$ & $\wedge$ & $1 / 29$ & 1 & $T / V I$ & 1 \\
\hline ir & $-\cdot / 4$. & r & r & 1 & $r / T$. & v & $1 / 1$. & r \\
\hline$\Delta$ & $-\cdot 1 \cdot \Delta$ & 9 & $r / 4 G$ & 9 & $1 / v 9$ & 9 & $1 / v$ & r \\
\hline 1 & $1 / V r$ & 14 & $r / 9)$ & r & $1 / 09$ & r & T/NT & r \\
\hline r & $1 / 41$ & v & r/VG & 1. & $1 / N \Delta$ & $r$ & $r / \cdot 1$ & $\Delta$ \\
\hline 9 & -.111 & $\Delta$ & $r / 19$ & $\Delta$ & r & $\Delta$ & $1 / 19$ & 4 \\
\hline r &.$- / 4 T$ & $\wedge$ & $r / \Delta \omega$ & 9 & $1 / 91$ & 11 & $1 / \Delta 9$ & v \\
\hline$\wedge$ & $-\cdot / r$. & r & ए/9६ & r & $r / \cdot \Lambda$ & 4 & $1 / M \Lambda$ & $\wedge$ \\
\hline 11 & $-\cdot / \pi \Delta$ & r & $r / . \varphi$ & ir & $1 / v$ & r & $1 / K F$ & 9 \\
\hline 9 & - & 11 & $r / 19$ & 11 & $1 / N \Delta$ & ir & $1 / 4 r$ & 1. \\
\hline 1. & $-\cdot / \mu r$ & 4 & $\Gamma / \Lambda \varphi$ & r & t/l. & $\wedge$ & $1 / V \Delta$ & 11 \\
\hline If & $-\cdot 109$ & 1. & $r / T F$ & v & $1 / 9$. & $1 f$ & $1 / K F$ & ir \\
\hline f & $-\cdot / \cdot \cdot r$ & ir & $r / 1 \Delta$ & If & $1 / \Delta V$ & 1. & $1 / \Delta \Lambda$ & Ir \\
\hline V &.$/ 1 T$ & F & ए/१६ & f & $r / . r$ & r & $1 / 94$ & If \\
\hline
\end{tabular}

جدول س. ماتريس اثر كل (H)

\begin{tabular}{|c|c|c|c|c|c|c|c|c|c|c|c|c|c|}
\hline $\begin{array}{c}2 \\
3 \\
3 \\
3 \\
2 \\
3 \\
13 \\
\frac{3}{3} \\
3 \\
3\end{array}$ & 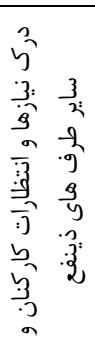 & $\begin{array}{l}\overline{3} \\
\overline{3} \\
\overline{3}\end{array}$ & $\frac{\bar{\alpha}}{2}$ & $\begin{array}{l}\frac{3}{3} \\
\frac{3}{3} \\
\frac{-2}{y} \\
\frac{1}{y}\end{array}$ & 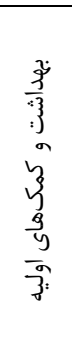 & 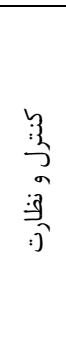 & 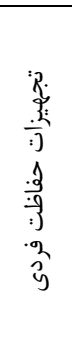 & $\begin{array}{l}3 \\
3 \\
3 \\
3 \\
3 \\
3 \\
3 \\
\frac{2}{3} \\
3\end{array}$ & 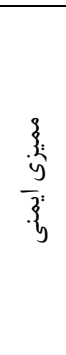 & 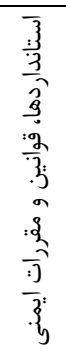 & $\begin{array}{c}3 \\
\vdots \\
: 3 \\
3 \\
3 \\
\stackrel{3}{3} \\
\frac{3}{5}\end{array}$ & $\begin{array}{l}3 \\
3 \\
3 \\
3 \\
5 \\
\vdots 3 \\
3 \\
3 \\
3 \\
3\end{array}$ & $\begin{array}{l}3 \\
3 \\
3 \\
3 \\
3 \\
3 \\
3 \\
y \\
y\end{array}$ \\
\hline$\cdot|\pi|$ &.$/ 1 \wedge$ & $\cdot \pi$. & $\cdot \pi r$ & .119 & .119 & . & $\cdot|r|$ & $\cdot|r|$ & .1 .1 & $\cdot / \cdot V$ & .119 &.$/ T T$ & $1 / 1 r$ \\
\hline.$/ 1 V$ & .111 & . & $\cdot / / V$ &.$/ 1 T$ & .111 & r & r &.$/ 19$ &.$/ 1 T$ & .1 .9 &.$/ T r$ & $1 / 1$. &.$/ T$ \\
\hline rו/. & .111 & . /IT & r/l. & .111 & .111 & .119 &.$/ 14$ & $\cdot / T$ & .111 &.$/ . r$ & $1 / \cdot V$ & $\cdot / I V$ & $\cdot / 10$ \\
\hline .119 & r/l & $\cdot / 1 \Delta$ & $\cdot \pi$. & $\cdot / 1 \Lambda$ &.$/ 1 F$ & $\cdot / r$. & .119 & .119 &.$/ 1 \wedge$ & $1 / . r$ & $\cdot / 11$ & $\cdot / r$. &.$/ 1 F$ \\
\hline.$/ 11$ &.$/ 1 T$ & rו/. & $\cdot / 1 \Lambda$ &.$/ 1 T$ &.$/ 1 T$ &.$/ 11$ & $\cdot / I V$ &.$/ 1 F$ & $1 / \cdot 9$ & .1 .9 & rון & $\cdot / 1 \Lambda$ & זו/. \\
\hline.$/ 1 F$ & .111 & r/l & $\cdot / / \gamma$ &.$/ 19$ &.$/ 1 T$ &.$/ 1 V$ & rו/. & $1 / 1$. &.$/ 1 T$ & .1 .4 & $\cdot / T r$ &.$/ 11$ &.$/ 1 T$ \\
\hline .119 & $\cdot / 1$. &.$/ 11$ &.$/ 1 T$ & .11 & $\cdot 11$ &.$/ 1 T$ & $1 / \cdot V$ & $\cdot / T$ & .11 &.$/ . T$ & $\cdot / 1$. & .119 &.$/ 11$ \\
\hline.$/ 1 F$ & .111 & r//. &.$/ 1 f$ &.$/ 1 T$ &.$/ 1 T$ & $1 / 1$. & $\cdot / I V$ & $\cdot / 1 \mathrm{~V}$ & .119 & / $/ . r$ & $\cdot / 1 \Delta$ & $\cdot / 1 \Lambda$ & $\cdot / 1 Y$ \\
\hline .111 & .1 .9 & $\cdot 11$. & .111 & .1 .9 & $1 / .9$ & .111 & .11. & .11. & .1 .9 &.$/ . r$ & .1 .9 & .111 & $\cdot 11$. \\
\hline .111 & .1 .9 &.$/ 1 F$ & .111 & $1 / .4$ & $\cdot / 1$. &.$/ 11$ & .111 &.$/ 11$ &.$/ 1$ &.$/ . r$ & $\cdot / 1$. & .111 & $\cdot / 1$. \\
\hline .119 & .111 &.$/ 1 T$ & $1 / .9$ & .111 & $\cdot / 1 \Delta$ & r//. &.$/ 19$ & .119 & .111 &.$/ . T$ &.$/ 11$ & זו/. & $\cdot / 1 T$ \\
\hline .111 & .1 .9 & 11.4 & .111 & .1 .9 & .1 .9 & .111 & $\cdot / 1$. & $\cdot / 1$. & .1 .9 &.$/ . r$ & .1 .9 & .111 & $\cdot / 1$. \\
\hline.$/ 14$ & $1 / .4$ & $\cdot / 10$ &.$/ 1 T$ & .111 &.$/ 1$ &.$/ 1 T$ & .111 & $\cdot / T$ & .111 &.$/ . r$ &.$/ 11$ &.$/ 1 T$ & $\cdot / \| f$ \\
\hline $1 / 1$. & 1119 &.$/ 1 \mathrm{~V}$ &.$/ 1 \mathrm{~V}$ &.$/ 1 T$ &.$/ T T$ &.$/ 1 \mathrm{~V}$ &.$/ 1 F$ & $\cdot / \| F$ &.$/ 1 T$ & .1 .9 &.$/ 1 T$ &.$/ 1 F$ & .119 \\
\hline
\end{tabular}

مى شود، مدل نهايى از جههار سطح تشكيل شده است؛ بايد

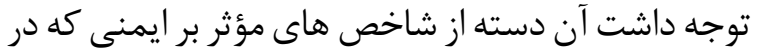

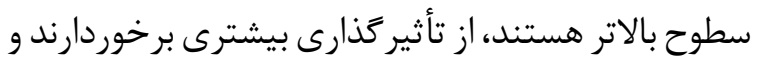

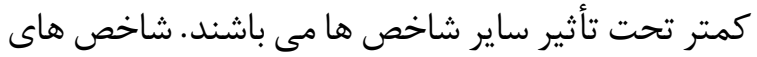

نهايى به صورت مدل سلسله مراتبى از شاخص هاى سطح

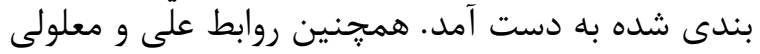
بين شاخص ها در اين مدل نشان داده شده است است (شكل

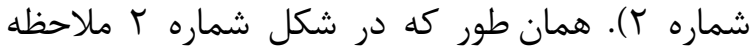


تجزيه و تحليل عوامل مؤثر بر عملكرد ايمنى...

جدول fا. ماتريس دستيابى (K)

\begin{tabular}{|c|c|c|c|c|c|c|c|c|c|c|c|c|c|c|}
\hline If & Ir & Ir & 11 & 1. & 9 & $\wedge$ & V & 9 & $\Delta$ & r & $r$ & $r$ & 1 & شاخص \\
\hline 1 & 1 & 1 & 1 & 1 & 1 & 1 & 1 & 1 & 1 & $\cdot$ & 1 & 1 & 1 & 1 \\
\hline . & . & . & . & . & . & . & . & . & . & . & . & 1 & . & $r$ \\
\hline . & . & . & . & . & . & . & . & . & . & . & 1 & . & . & $r$ \\
\hline . & . & . & 1 & 1 & . & 1 & 1 & 1 & 1 & 1 & 1 & 1 & . & r \\
\hline 1 & . & . & 1 & . & . & 1 & . & . & 1 & . & . & 1 & . & $\Delta$ \\
\hline . & . & . & . & . & . & . & . & 1 & . & . & . & 1 & . & 9 \\
\hline . & . & . & . & . & . & . & 1 & . & . & . & . & . & . & v \\
\hline . & . & . & . & . & . & 1 & . & . & . & . & . & 1 & . & $\wedge$ \\
\hline . & . & . & . & . & 1 & . & . & . & . & . & . & . & . & 9 \\
\hline . & . & . & . & 1 & . & . & . & . & . & . & . & . & . & 1. \\
\hline . & . & . & 1 & . & . & . & . & . & . & . & . & . & . & 11 \\
\hline . & . & 1 & . & . & . & . & . & . & . & . & . & . & . & Ir \\
\hline . & 1 & . & . & . & . & . & . & . & . & . & . & . & . & $1 \pi$ \\
\hline 1 & . & . & . & . & . & . & . & . & . & . & . & . & . & If \\
\hline
\end{tabular}

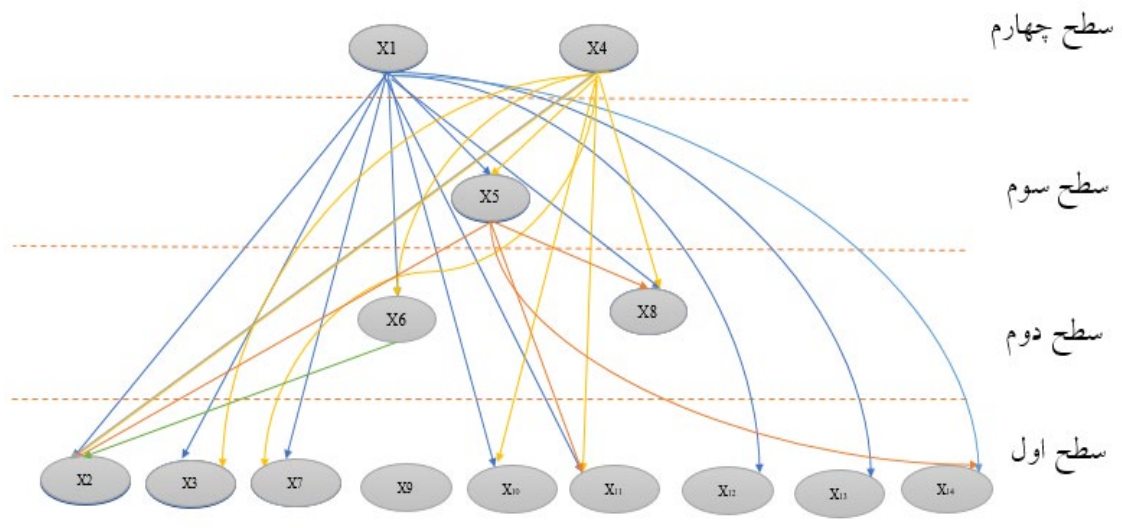

شكل r. سطح بندى شاخص هاى مؤثر بر ايمنى

نيروى برق بايد به شاخص هاى ايمنى كه نقش علت دارند

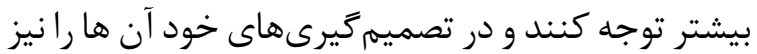

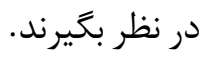
شاخص هاى مؤثر بر ايمنى با توجه به ميزان قدرت

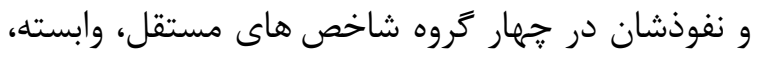

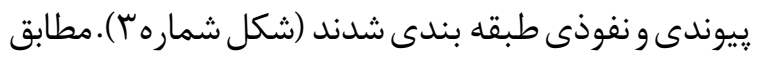

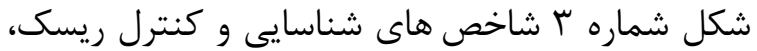

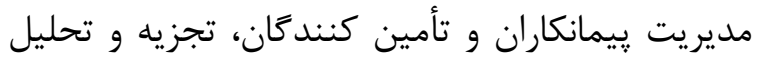

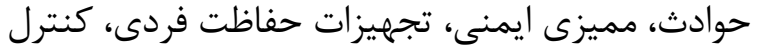

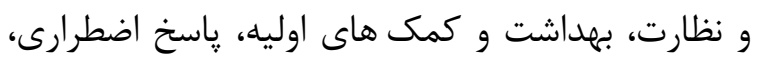

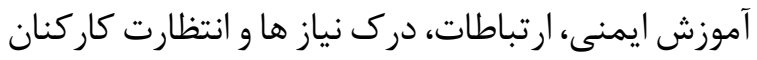

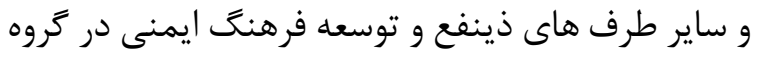

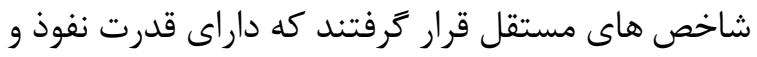
وابستخى كمى هستند. علاوه بر اين، شاخص هاى ترفي تعهد
تعهد مديريت عالى و استاندارد ها، قوانين و مقر رات ايمنى داراى بيشترين قدرت اثر كذارى بر روى سايت ساير شاخص هاني

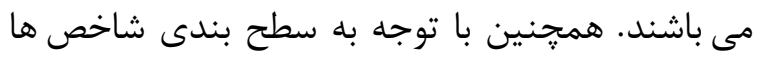

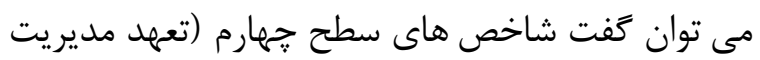

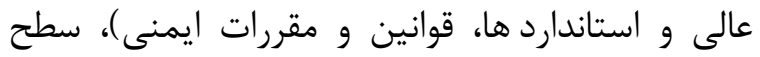

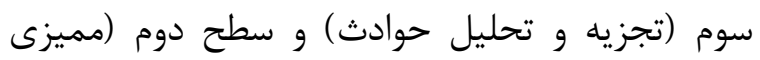

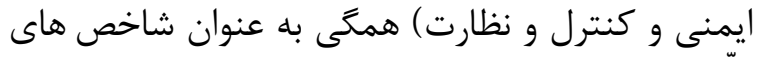

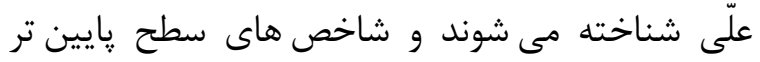
يعنى سطح اول (شناسايى و كنترل ريسك، مدئ مديريت

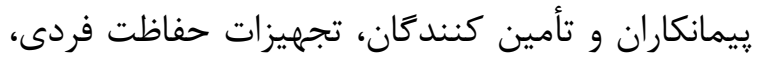

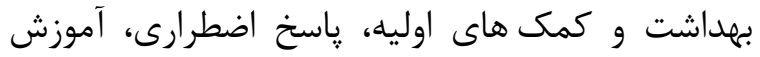

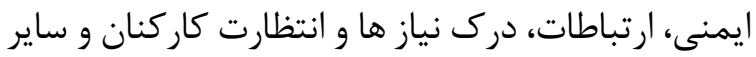

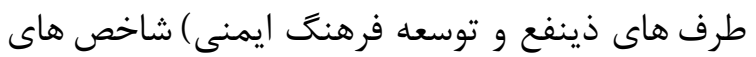

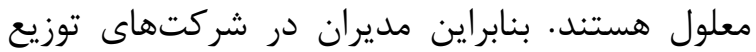

Iran Occupational Health. 2021 (01 June);18: 12 


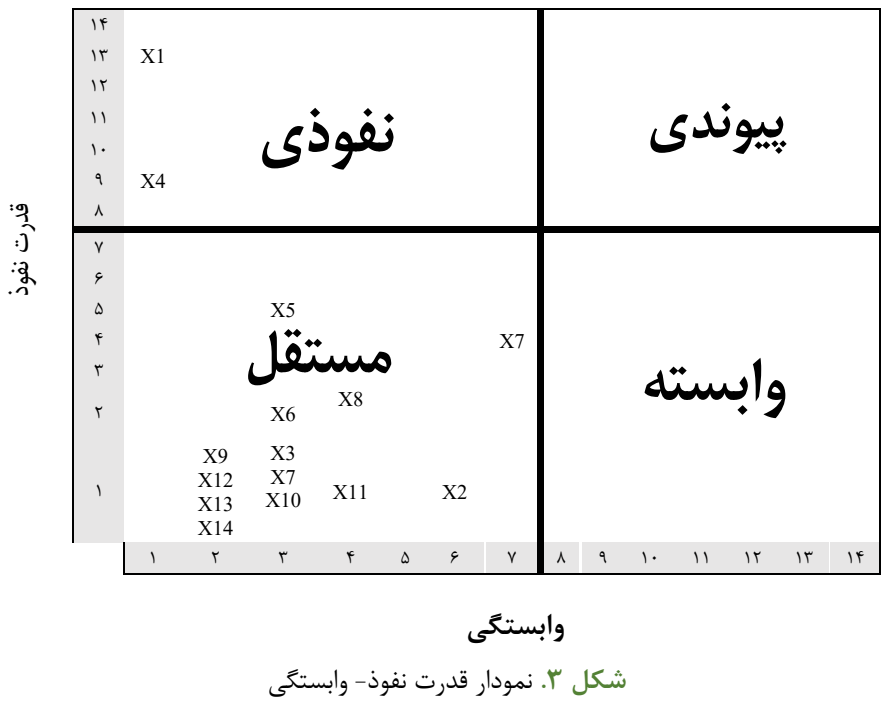

در سه دسته اصلى سازمانى، فنى و انسانى طبقه بندى

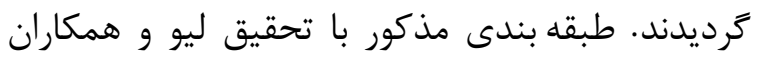

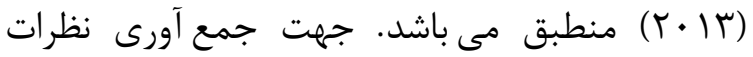

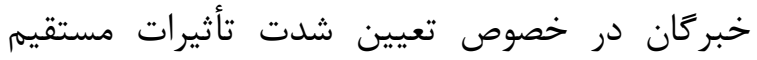
بين شاخص ها از يرسشنامه ديمتل استفاده كرديد. بان

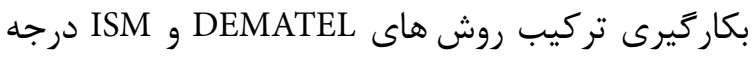

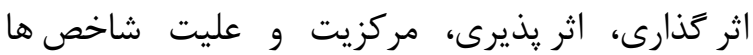

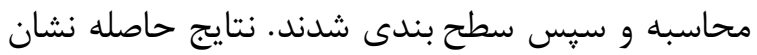

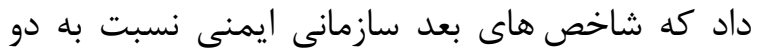

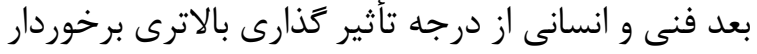

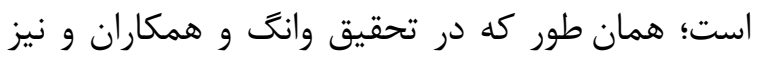

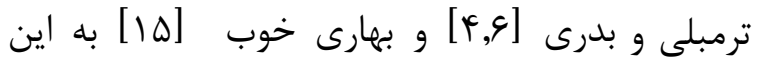

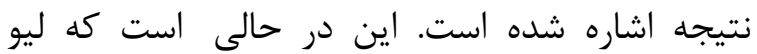

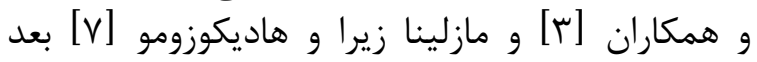

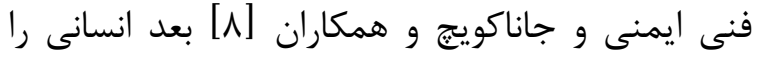

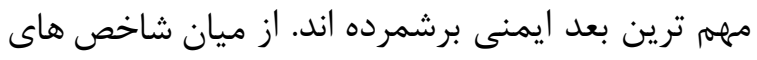

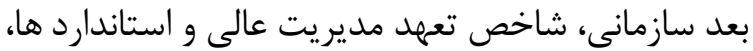

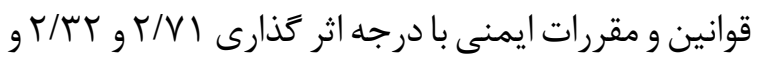

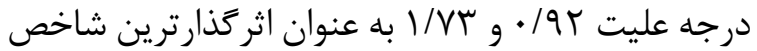

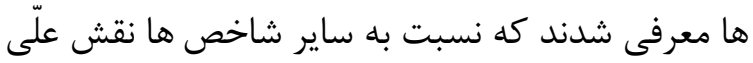

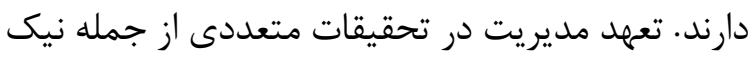

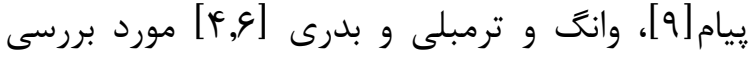

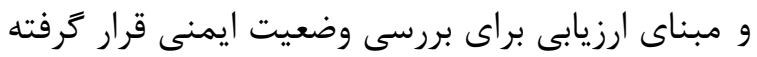

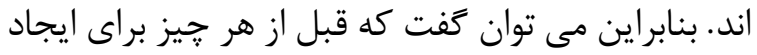

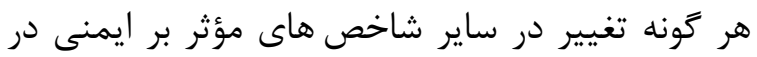

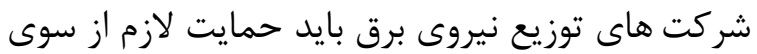

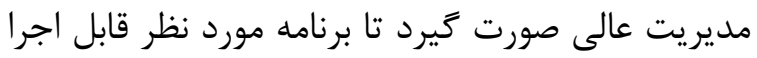
و اثربخش باشد. مديريت عالى مى تواند با تعريف خط مُ نط
مديريت عالى و استاندارد ها،

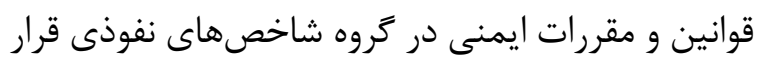

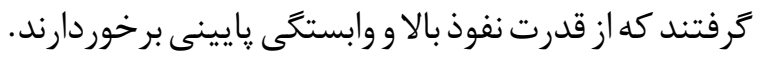

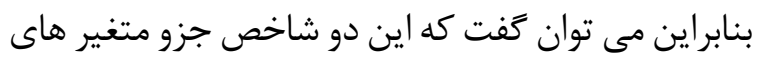
كليدى محسوب مىشوند.

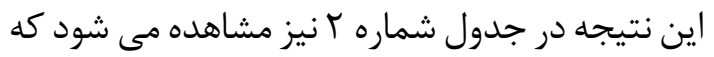

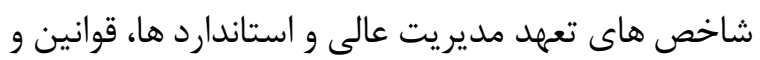

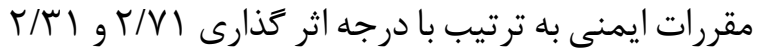

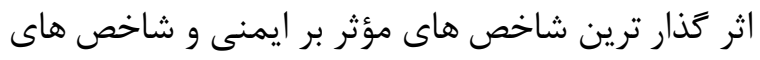

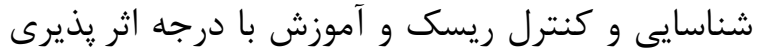

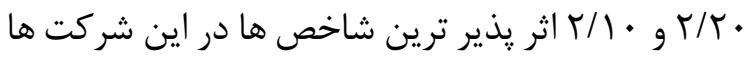

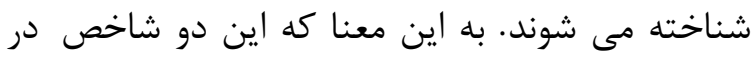

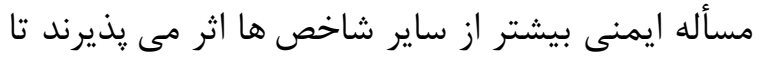

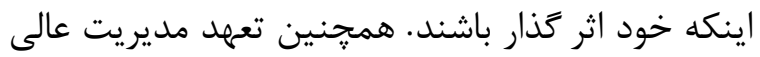

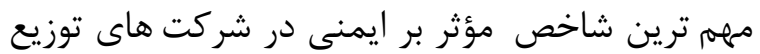

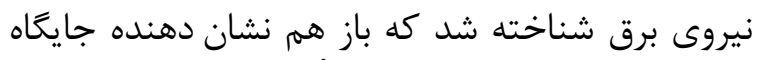

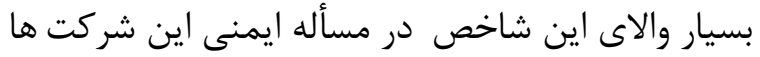

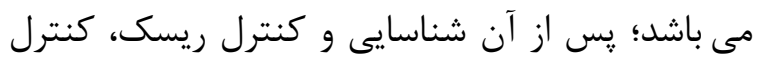

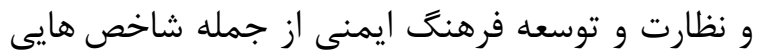

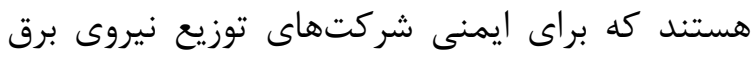
بسيار مهرم تلقى شدند.

$$
\text { بحث و نتيجه كَيرى }
$$
در تحقيق حاضر به بررسى عوامل مؤثر بر ايمنى در دري

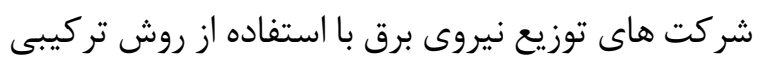

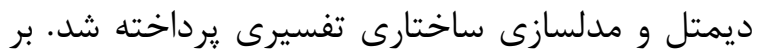

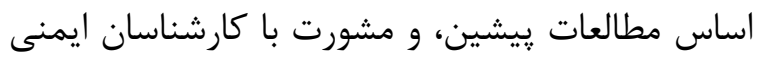

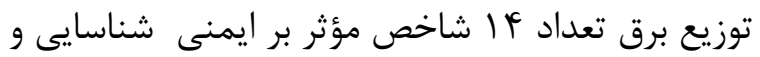


تجزيه و تحليل عوامل مؤثر بر عملكرد ايمنى.

Company, [Thesis]. Marvdasht: Humanity Science of Islamic Azad University 2017 (Persian).

[2] Rahmani S, Omidvari M. Assessing safety riskin electricity distribution processes using ET \& BA improved technique and its ranking by VIKOR and TOPSIS models in fuzzy environment. Journal of Health and Safety at Work. 2016; 6 (1): 1-12 (Persian).

[3] Liu Y, Chen J, Cheng Sh, Hsu M, Wang Ch. Evaluation of Safety Performance in Process Industries. Wiley Online Library 2013.

[4] Wang L, Cao Q, Zhou L. Research on the influencing factors in coal mine production safety based on the combination of DEMATEL and ISM. Safety Science 2018; 103: 51-61.

[5] Azar A, Khosravani F, Jalali R. soft operation Research -problem structuring. Tehran: Industrial Management Institute; 2017 (Persian).

[6] Tremblay A Badri A. A novel tool for evaluating occupational health and safety performance in small and medium-sized enterprises: The case of the Quebec forestry/pulp and paper industry. Safety Science 2018; 101: 282-294

[7] Mazlina Zaira M, Hadikusumo B. Structural equation model of integrated safety intervention practices affecting the safety behaviour of workers in the construction industry. Safety Science 2017; 98: 124135.

[8] Janackovic G, Stojiljkovic E, Grozdanovic M. Selection of key indicators for the improvement of occupational safety system in electricity distribution companies. Safety Science 2017.

[9] Nikpayam R. Safety Culture Assessment in Mashhad Power Distribution Company Based on RSSB Model. 29th International Power System Conference. Tehran 2014 (Persian).

[10] Rezapour M. Proposing a Framework for Safety Assessment in Power Distribution Companies - Soft Operations Research Approach (Case Study: Khorasan Razavi Province Power Distribution Company), [Thesis]. Tehran: Humanity Science of Shahed university; 2019 (persian).

[11] Hajininezhad Z. Work Accident. 2017. Available at:http://www.irna.ir/fa/News/82769687. September 18, 2017 (persian).

[12] barghnews. Iran electrocution Statistics. 2015; [1]. Available athttp://barghnews.com/fa/news /10575. November 19, 2017 (persian).

[13] Santos J, Miguel-Dávila, J, Herrera L, Nieto M. Safety Management System in TQM environments. Safety Science. 2018; 101: 135-143.

[14] Rahmanim A, Khadem M, Madreseh E, Aghaei H, Raei M, Karchani M. Descriptive Study of Occupational
مشى ايمنى بر اساس سيستم مديريت ايمنى و بهداشت

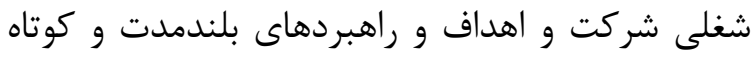

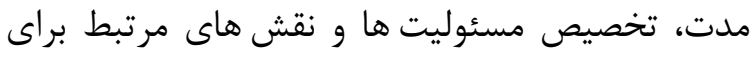
يياده سازى اين سيستم و تدوين برنامه های هاى عملياتى در

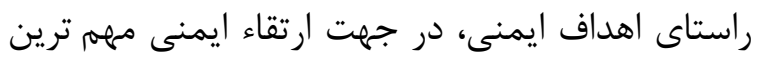

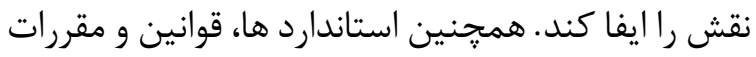
ايمنى بايستى بله طور كامل و روشن به همه كاركنان و ساير ذينفعان شركت اطلاع رسانى شود و با نظارت سخت سته

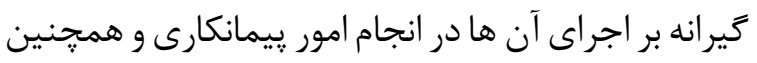

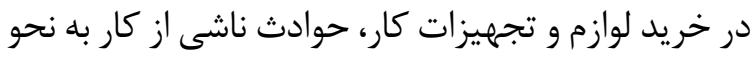

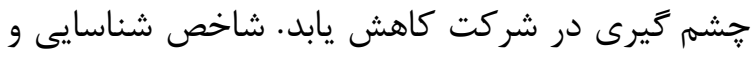

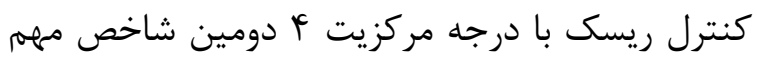

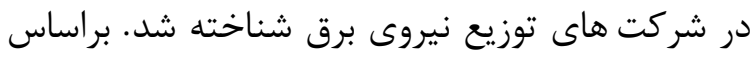

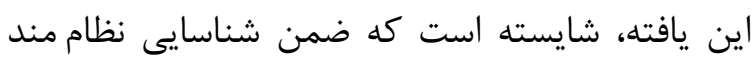

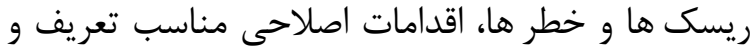

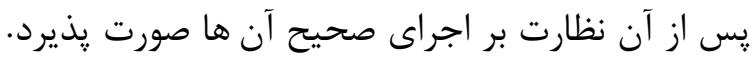

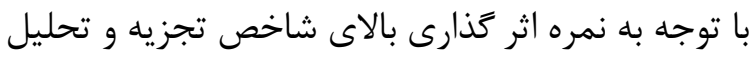

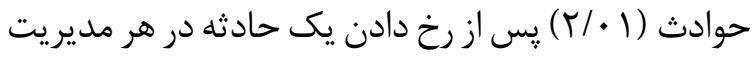

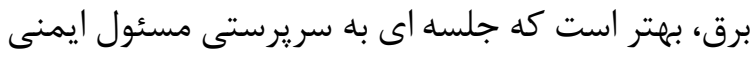

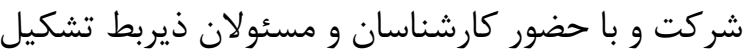

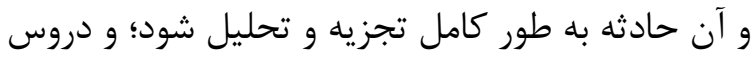

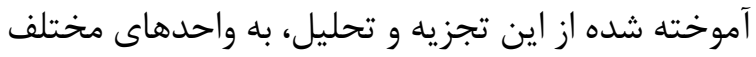

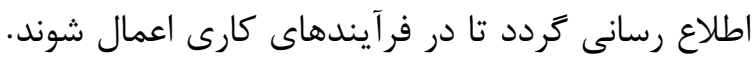

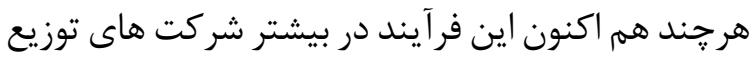

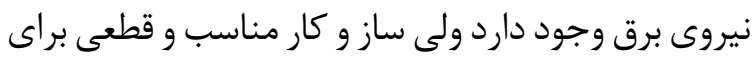

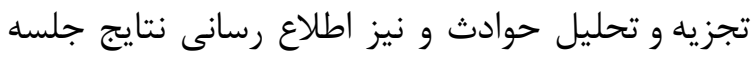

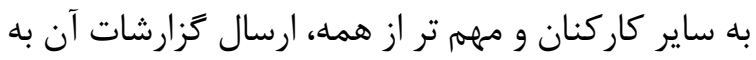

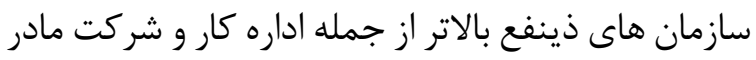

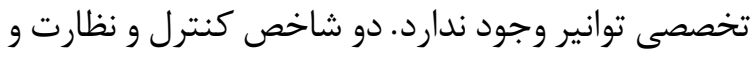

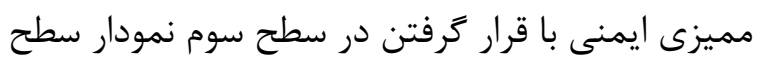

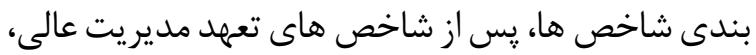

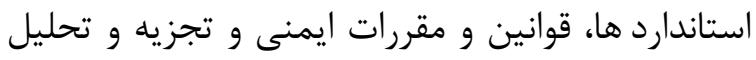

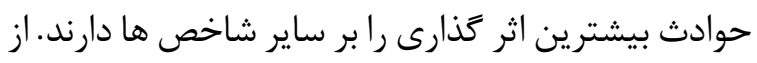

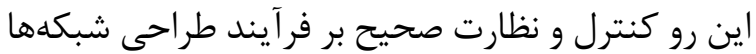

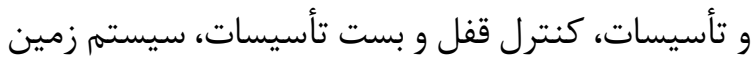

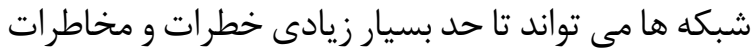

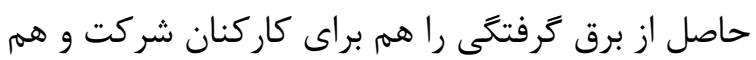

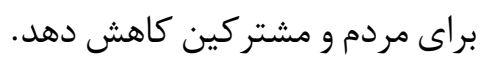

من

[1] GhadiriJafarBeigLu B. Identifying and Factors Prioritizing Influencing Safety Culture Using AHP Technique in Fars province Power Distribution 
Balanced Scorecard Method, [Thesis].Gilan: Literature and Humanity Science of Gilan university (persian).

[27] Naseri A, Sepehri M, Mahmoudi S. Strategic performance evaluation of Health, Safety and Environment (HSE) based on Balanced Scorecard (BSC), the case study of a corporation in energy industry. Iran Occupational Health. 2014; 11 (1) :79-94 (persian).

[28] Yarahmadi R, shahkohi F, taheri F, moridi P. Priority of Occupational Safety and Health indexes Based on the Multi Criteria Decision Making in Construction Industries . Iran Occupational Health. 2016; 12 (6) :39-47(persian).

[29] Gunduz M, Ahsan B. Construction safety factors assessment through Frequency Adjusted Importance Index. International Journal of Industrial Ergonomics. 2018; 64:155-162.

[30] Khalili Z.Designing a Model for Performance Evaluation of Health, Safety and Environment Management System, [Thesis]. Engineering of Kharazmi University; 2012 (persian).

[31] Valianpour F. Performance Evaluation of Gas Transmission System Based on Human and Safety Indicators Using combined Mathematical and Statistical Methods, [Thesis]. Tafresh: Industrial Engineering of Tafresh University (persian).

[32] Hakimikhah A. Occupational and Environmental Health and Safety Risk Assessment for the Construction Project and Installation of a Distillation Unit for the Persian gulf Stars Gas Condensate Refinery in Bandar Abbas by FMEA, [Thesis]. Tehran:Agricultural Engineering of Payame Noor University; 2015 (persian).

[33] Inan U, Gül S,Yılmaz H. A multiple attribute decision model to compare the firms' occupational health and safety management perspectives. Safety Science. 2017; 91: 221-231.

[34] Beriha G, Patnaik B, Mahapatra S, Padhee S. Assessment of safety performance in Indian industries using fuzzy approach. Expert Systems with Applications. 2012; 39: 3311-3323.

[35] Gunduz M, Birgonul M, Ozdemir M. Development of a safety performance index assessment tool by using a fuzzy structural equation model for construction sites. Automation in Construction. 2018; 85: 124-134.

[36] Muniz B, Manuel J and Vazquez-Ordas C. Relation between occupational safety management and firm performance. Safety Science. 2009; 47: 980-991.

[37] Mullen J, Kelloway K, Teed M. Employer safety obligations, transformational leadership and their interactive effects on employee safety performance. Safety Science. 2017; 91: 405-412.

[38] Xia N, Xueqing W, Xing L, Dan W. Is there agreement
Accidents and their Causes among Electricity Distribution Company Workers at an Eight-year Period in Iran. Safety and Health at Work. 2013; 4: 160-165.

[15] Baharikhoob S.Identification and Ranking of Critical Factors Affecting Industrial Safety (Case Study: Shiraz Power Distribution Company) [Thesis]. Marvdasht: Educational Science of Islamic Azad University (persian).

[16] Yiu N, Sze N, Chan D. Implementation of safety management systems in Hong Kong construction industry - A safety practitioner's perspective. Journal of Safety Research. 2018; 64: 1-9.

[17] Karanikas N. Evaluating the horizontal alignment of safety management activities through cross-reference of data from safety audits, meetings and investigations. Safety Science. 2017; 98: 37-49.

[18] British Standards Institution. OHSAS 18001:2007-Occupational Health and Safety Assessment Series-Requirements. 2007.

[19] Asgharizade E, Ghasemi A, Behrouz M. Accident Evaluation, Based on Safety Performance Indicators, Using EVAMIX- Case Study: Natural Gas Pipelines. Journal of Crisis Management.2014; 3(1):57-63 (persian).

[20] Barkhordari A, Dehghani A, Kianfar A, Mahmoudi S, Aminifard F. Safety performance evaluation using proactive indicators in a selected industry. Journal of Occupational Health Engineering. 2015; 1 (4) :49-59 (persian).

[21] Goodarzi R, Arghami S, Pouyakian M. Identification of factors affecting safety culture in Iranian thermal power plants. Journal of Occupational Hygiene Engineering. 2016; 3 (2) :12-20 (persian).

[22] Dormohammadi A, Mohammadfam I, Zarei E. Presentation of a practical framework for performance assessment of HSE in construction contractors. Iran Occupational Health. 2017; 13 (6) :10-22(persian).

[23] Mohammadfam I, Kamalinia M, Momeni M, et al. Evaluation of the quality of occupational health and safety management systems based on key performance indicators in certified organizations. Safety and Health at Work. 2016;8(2): 156-161.

[24] Mohammadfam I, Kamalinia M, Golmohammadi R, Momeni M, Hamid Y, Soltanian A. A framework for evaluating the performance of OHSMSs using multicriteria methods. Iran Occupational Health. 2017; 14 (1) :23-36 (persian).

[25] Chmiel N, Laurent J, Hansez, I. Employee perspectives on safety citizenship behaviors and safety violations. Safety Science. 2017; 93: 96-107.

[26] Kouchakkosari S.Performance Evaluation of Health, Safety and Environment Management System Using 
تجزيه و تحليل عوامل مؤثر بر عملكرد ايمنى...

between safety leading indicators and safety climate levels. Journal of Safety Research. 2017; 62: 23-32.

[40] Memariani A, Azar A.AHPA New Technique for Group Decision Making. Management knowledge. 1995; 27, 28: 22-32 (persian). between worker self and supervisor assessment of worker safety performance? An examination in the construction industry. Journal of Safety Research. 2018; 65: 29-37.

[39] Givehchi S, Hemmativaghef E, Hoveidi H. Association 